\title{
On a non-linear 2D fractional wave equation
}

\author{
Aurélien Deya* \\ ${ }^{a}$ Institut Elie Cartan, University of Lorraine B.P. 239, 54506 Vandoeuvre-lès-Nancy, Cedex France \\ E-mail: *aurelien.deya@univ-lorraine.fr
}

Abstract. We investigate the following non-linear stochastic wave equation model:

$$
\left\{\begin{array}{l}
\partial_{t}^{2} u-\Delta u=u^{2}+\dot{B}, \quad t \in[0, T], x \in \mathbb{R}^{2} \\
u(0, .)=\phi_{0}, \partial_{t} u(0, .)=\phi_{1}
\end{array}\right.
$$

where $\phi_{0}, \phi_{1}$ are deterministic initial conditions in an appropriate Sobolev space and $\dot{B}$ stands for a space-time fractional noise. In this two-dimensional situation, we develop a strategy based on a third-order expansion of the equation, which, combined with a Wick-renormalization procedure, allows us to extend the results of [1] to a rougher noise.

We also point out the limits of this specific strategy when considering a highly rough noise.

Résumé. Nous nous intéressons au modèle d'équation des ondes stochastique non-linéaire suivant:

$$
\left\{\begin{array}{l}
\partial_{t}^{2} u-\Delta u=u^{2}+\dot{B}, \quad t \in[0, T], x \in \mathbb{R}^{2} \\
u(0, .)=\phi_{0}, \partial_{t} u(0, .)=\phi_{1}
\end{array}\right.
$$

où $\phi_{0}, \phi_{1}$ sont des conditions initiales déterministes dans un espace de Sobolev approprié et $\dot{B}$ représente un bruit fractionnaire espace-temps. Dans cette situation bi-dimensionnelle, notre stratégie est basée sur un développement de l'équation à l'ordre trois, qui, combiné à une procédure de renormalisation de type Wick, nous permet d'étendre les résultats de [1] à des bruits plus rugueux.

Nous mettons également en avant les limites de cette stratégie particulière en présence de processus très irréguliers. Keywords: Stochastic wave equation, Fractional noise, Wick renormalization.

\section{Introduction and main results}

Consider the following non-linear stochastic wave model:

$$
\left\{\begin{array}{l}
\partial_{t}^{2} u-\Delta u=u^{2}+\dot{B}, \quad t \in[0, T], x \in \mathbb{R}^{d} \\
u(0, .)=\phi_{0}, \partial_{t} u(0, .)=\phi_{1}
\end{array}\right.
$$

where $\phi_{0}, \phi_{1}$ are (deterministic) initial conditions in an appropriate Sobolev space and $\dot{B}$ stands for a (rough) stochastic noise. In many situations (that include the space-time white noise case for $d \geq 2$ ), we know that the solution of the associated "homogeneous" equation, i.e. the solution of

$$
\left\{\begin{array}{l}
\partial_{t}^{2} \Psi-\Delta \Psi=\dot{B}, \quad t \in[0, T], x \in \mathbb{R}^{d} \\
\Psi(0, .)=0, \partial_{t} \Psi(0, .)=0
\end{array}\right.
$$


can only be defined as a distribution (in space), which immediately gives us an idea of how difficult the interpretation and the treatment of the additional product term $u^{2}$ in (1). In the white-noise situation and when considering the equation on a torus, these difficulties have been recently overcome by Gubinelli, Koch and Oh, first for $d=2[3]$ and then for $d=3$ [4]. Their strategy involves a deterministic expansion of the equation, together with the stochastic construction of some suitable "iterated integrals" (above the white noise), at the core of the model dynamics.

In the continuity of our previous study [1], we here propose to offer more flexibility as far as the noise model is concerned, by extending the analysis to a space-time fractional noise. To be more specific, we will focus in this paper on the two-dimension case (i.e., $d=2$ in (1)) and consider a noise $\dot{B}:=\partial_{t} \partial_{x_{1}} \partial_{x_{2}} B$ defined as the space-time derivative (in the sense of distributions) of a fractional Brownian sheet $B$ :

Definition 1.1. Let $(\Omega, \mathcal{F}, \mathbb{P})$ be a complete filtered probability space. For $H=\left(H_{0}, H_{1}, H_{2}\right) \in(0,1)^{3}$, we call a space-time fractional Brownian motion (or a fractional Brownian sheet) of Hurst index $H$ any centered Gaussian process $B: \Omega \times\left([0, T] \times \mathbb{R}^{2}\right) \rightarrow \mathbb{R}$ whose covariance function is given by

$$
\mathbb{E}\left[B_{s}\left(x_{1}, x_{2}\right) B_{t}\left(y_{1}, y_{2}\right)\right]=R_{H_{0}}(s, t) R_{H_{1}}\left(x_{1}, y_{1}\right) R_{H_{2}}\left(x_{2}, y_{2}\right),
$$

where

$$
R_{H_{i}}(x, y) \triangleq \frac{1}{2}\left(|x|^{2 H_{i}}+|y|^{2 H_{i}}-|x-y|^{2 H_{i}}\right) .
$$

At this point, we must recall that the first step of this analysis has been carried out in [1], where, thanks to a second-order expansion of the equation, we have been able to cover the case

$$
H_{0}+H_{1}+H_{2}>\frac{5}{4} .
$$

Our aim here is to go one step further and treat the situation where

$$
1<H_{0}+H_{1}+H_{2} \leq \frac{5}{4},
$$

a condition which will turn out to be optimal (at least when using our "Wick" expansion strategy, see Proposition 1.4 and Remark 1.10 below). Beyond the extension result itself, the study will give us the opportunity to settle a third-order procedure, which somehow generalizes the so-called Da Prato-Debussche trick and involves the construction of sophisticated third-order stochastic objects. As far as we know, such a third-order expansion, inspired by the recent developments in the parabolic setting (see e.g. [7, Section 1.1]), is new in the stochastic wave literature. In fact, while we were revising the present paper, a similar thirdorder-expansion strategy has been developed by Gubinelli, Koch and Oh in [4] for the three-dimensional and white-noise-driven version of (1). Their study additionally appeals to sophisticated considerations related to paraproducts, which will not be needed in the subsequent (two-dimensional) analysis.

In order to illustrate our strategy, and also to understand the whole difficulty raised by the transition from condition (2) to condition (3), let us start with a few heuristic considerations on the quadratic fractional model (1).

\subsection{Heuristic considerations}

For the sake of clarity, the following shortcut notations will be used throughout the paper: for all $T>0$, $\alpha \in \mathbb{R}$ and $p, q \geq 1$, we set

$$
L_{T}^{q} \mathcal{W}^{\alpha, p} \triangleq L^{q}\left([0, T] ; \mathcal{W}^{\alpha, p}\left(\mathbb{R}^{2}\right)\right) \quad, \quad L_{T}^{q} \mathcal{W}_{l o c}^{\alpha, p} \triangleq L^{q}\left([0, T] ; \mathcal{W}_{\text {loc }}^{\alpha, p}\left(\mathbb{R}^{2}\right)\right),
$$

where $\mathcal{W}^{\alpha, p}\left(\mathbb{R}^{2}\right)$ stands for the classical Sobolev space and

$\mathcal{W}_{\text {loc }}^{\alpha, p}\left(\mathbb{R}^{2}\right) \triangleq\left\{u \in \mathcal{S}^{\prime}\left(\mathbb{R}^{2}\right):\right.$ for every smooth compactly-supported function $\left.\rho: \mathbb{R}^{2} \rightarrow \mathbb{R}, \rho u \in \mathcal{W}^{\alpha, p}\left(\mathbb{R}^{2}\right)\right\}$. 
The related (space-time) norms will be denoted by $\mathcal{N}\left[. ; L_{T}^{q} \mathcal{W}^{\alpha, p}\right]$.

Besides, we will denote by $*_{x}$, resp. $*_{t, x}$, any convolution with respect to the space variable $x \in \mathbb{R}^{2}$, resp. the space-time variable $(t, x) \in \mathbb{R}^{3}$. Finally, we will use the convention $\mathcal{F}_{x}(\varphi)(\xi) \triangleq \int_{\mathbb{R}^{2}} d x e^{-\imath\langle\xi, x\rangle} \varphi(x)$ for the Fourier transform of any (suitable) function $\varphi: \mathbb{R}^{2} \rightarrow \mathbb{R}$.

At a formal level, the mild form of Equation (1) (with $d=2$ ) is given by

$$
u=\partial_{t}\left(G_{t} *_{x} \phi_{0}\right)+G_{t} *_{x} \phi_{1}+G *_{t, x} u^{2}+?,
$$

where $G$ stands for the wave kernel in $\mathbb{R}^{2}$, characterized by its Fourier transform

$$
\mathcal{F}_{x}\left(G_{t}\right)(\xi)=\frac{\sin (t|\xi|)}{|\xi|}, \quad t \geq 0, \xi \in \mathbb{R}^{2},
$$

and the symbol ? refers to the associated "homogeneous" solution, that is the solution of

$$
\left\{\begin{array}{l}
\partial_{t}^{2} \stackrel{\varphi}{\varphi}-\Delta ?=\dot{B} \quad, \quad t \in[0, T], x \in \mathbb{R}^{2} \\
\stackrel{\varphi}{ }(0, .)=0, \quad \partial_{t} \stackrel{\varphi}{ }(0, .)=0
\end{array}\right.
$$

Of course, due to the roughness of the noise $\dot{B}$, the interpretation of the latter equation is not exactly a standard issue: in fact, for a proper definition of ?, we will rely in the sequel on a specific approximation procedure (see the convergence statement for the first component in Proposition 1.3). As a result of this procedure, ? will (almost surely) appear as a stochastic process with values in the space $L_{T}^{\infty} \mathcal{W}_{l o c}^{-\alpha, p}$, for all $p \geq 2$ and $\alpha>\frac{3}{2}-\left(H_{0}+H_{1}+H_{2}\right)$. With condition (3) in mind, let us assume from now on that $\alpha>0$.

Going back to (5), a first natural idea toward a possible fixed-point argument (often referred to as the Da Prato-Debussche trick) is to consider the dynamics satisfied by the difference process $v \triangleq u-?$. To this end, we can rewrite the equation as

$$
v=\partial_{t}\left(G_{t} *_{x} \phi_{0}\right)+G_{t} *_{x} \phi_{1}+G *_{t, x} v^{2}+2 G *_{t, x}(v \cdot ?)+G *_{t, x}(?)^{2},
$$

and then try to identify some possible stable space for $v$. Observe however that we must here face with a new interpretation issue. Indeed, as ? is expected to take values in some negative-order Sobolev space, it is not clear to know a priori how we must interpret the product (?) ${ }^{2}$ in (7). This problem has been treated in [3] for the white-noise situation $H_{0}=H_{1}=H_{2}=\frac{1}{2}$, and then in [1] under the more general condition $H_{0}+H_{1}+H_{2}>\frac{5}{4}$. In both of these references, it is shown that (?) ${ }^{2}$ can only be understood in some Wick sense, that is through a renormalization procedure and the use of stochastic estimates, and the construction gives birth to a process with values in $L_{T}^{\infty} \mathcal{W}_{l o c}^{-2 \alpha, p}$ (a.s.). A first new result of the present study will consist in extending this property under condition (3) (see the convergence result for the second component in Proposition 1.3). Let us henceforth denote by

$$
\mathrm{Q} \rho \in L_{T}^{\infty} \mathcal{W}_{l o c}^{-2 \alpha, p}
$$

the resulting Wick interpretation of the product (?) ${ }^{2}$, and consider the related equation

$$
v=\partial_{t}\left(G_{t} *_{x} \phi_{0}\right)+G_{t} *_{x} \phi_{1}+G *_{t, x} v^{2}+2 G *_{t, x}(v \cdot ?)+G *_{t, x} \text { ९. . }
$$

At this point, recall that, even if the property is less convenient to handle than its parabolic counterpart, convolution with the wave kernel $G$ still yields some regularizing effect, as summed up through the so-called Strichartz inequalities (see e.g. [1, Propositions 3.2 and 3.3]). According to these results and using the symbol of for the convolution $G *_{t, x}$ \&, , we can (at least morally) expect to have

$$
\stackrel{Q}{Q} \in L_{T}^{\infty} \mathcal{W}_{l o c}^{1-2 \alpha, 2}
$$


Actually, just as with ? and 9, , the use of stochastic arguments will allow us to improve this regularity property and derive (see the third component in Proposition 1.3) that

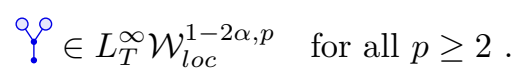

Going back to equation (9), we can then expect the solution $v$ to inherit the regularity of ? , so that, if we refer to standard distribution-theory results (such as those in the subsequent Proposition 2.2), the product $v \cdot ?$ involved in the equation is likely to make sense as long as $(1-2 \alpha)+(-\alpha)>0$, that is as long as $\alpha \in\left(0, \frac{1}{3}\right)$. These heuristic arguments somehow account for the success of the "second-order" expansion used in $[1,3]$ (in both references, it is assumed that $\alpha \in\left(0, \frac{1}{4}\right)$ ).

In order to go one step further and handle the situation where $\alpha \in\left(\frac{1}{3}, \frac{1}{2}\right)$ (which encompasses condition (3)), let us iterate the above principles and consider the new process $w \triangleq v-$ ?. Equation (9) now turns into

$$
\begin{aligned}
& w=\partial_{t}\left(G_{t} *_{x} \phi_{0}\right)+G_{t} *_{x} \phi_{1}+G *_{t, x}(w+?)^{2}+2 G *_{t, x}((w+?) ?) \\
& =\partial_{t}\left(G_{t} *_{x} \phi_{0}\right)+G_{t} *_{x} \phi_{1}+G *_{t, x} w^{2}+G *_{t, x}\left(\varphi^{q}\right)^{2}+2 G *_{t, x}(w \cdot \varphi)+2 G *_{t, x}(w \cdot \varphi)+2 G *_{t, x} \varphi_{\ell},
\end{aligned}
$$

where the "third-order" process $\because \%$ is defined as

$$
q \triangleq q \cdot ? .
$$

Again, the interpretation of the latter product is not clear when $\alpha \in\left(\frac{1}{3}, \frac{1}{2}\right)$ (since $\left.(1-2 \alpha)+(-\alpha)<0\right)$, but, just as with ๑९, we can hope for a "stochastic construction" of this product, leading (a.s.) to a well-defined element

$$
q \in L_{T}^{\infty} \mathcal{W}_{l o c}^{-\alpha, p}
$$

The construction of this process above the fractional noise will be one of the main technical results of our study (it corresponds to the convergence property of the fourth component in Proposition 1.3 below). Once endowed with $\%$, and along the same pattern as above, we can (morally) expect to have

$$
w \in L_{T}^{\infty} \mathcal{W}_{l o c}^{1-\alpha, 2}
$$

so that the product $w \cdot ?$ in $(11)$ can make sense for any $\alpha \in\left(\frac{1}{3}, \frac{1}{2}\right)$ (due to $(1-\alpha)+(-\alpha)>0$ ), leading finally to the possibility of a well-posed equation in our setting. The details of this deterministic procedure will be the topic of Section 2.2 below.

To end with these heuristic considerations, we need to specify that, since the controls in (8)-(10)-(12) are only local, we can only aim at a local solution in this study, in time as well as in space (say on the fixed space domain $\{|x| \leq 1\}$ ). For a clear expression of this restriction, let us thus introduce, for the rest of the paper, a smooth function $\rho: \mathbb{R}^{2} \rightarrow \mathbb{R}$ with support included in $\{|x| \leq 2\}$ such that $\rho(x)=1$ for $|x| \leq 1$, and note that we will rather consider the following "localized" version of (11):

$$
\begin{aligned}
w= & \partial_{t}\left(G_{t} *_{x} \phi_{0}\right)+G_{t} *_{x} \phi_{1} \\
& +G *_{t, x}\left(\rho^{2} w^{2}\right)+G *_{t, x}\left(\rho^{2}(\varrho)^{2}\right)+2 G *_{t, x}((\rho w) \cdot(\rho !))+2 G *_{t, x}((\rho w) \cdot(\rho !))+2 G *_{t, x}\left(\rho^{2} \varrho_{\ell}\right) .
\end{aligned}
$$

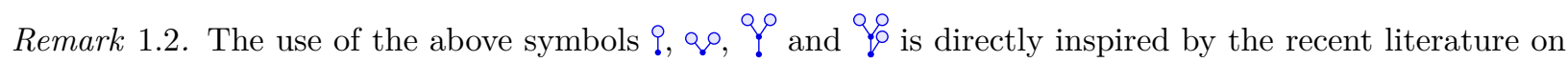
parabolic SPDEs (see e.g. [6, Section 4] or [7, Section 1]). In fact, as the reader may have guessed it, the construction of these symbols (morally) obeys the following simple rules: $(i)$ the circle symbol $\circ$ refers to the noise $\dot{B}$; (ii) each line ! corresponds to a convolution with the wave kernel $G$; (iii) two (sub-)symbols attached to a same black knot are just multiplied between each other (that is, the associated processes are multiplied between each other). Note however that the latter "code" is only heuristic: in particular, it does not take the possible renormalization procedures into account (as the one involved in the "Wick" definition of $\left.Q_{p}\right)$. 


\subsection{Stochastic constructions}

As evoked earlier, a proper definition of the stochastic components

$$
(?, \text { Q०, ?, , : }
$$

at the center of the above analysis will be obtained through an approximation procedure. Namely, starting from some smooth approximation $\dot{B}^{n}$ of the fractional noise, we first observe that, for each fixed $n \geq 1$, the homogeneous equation

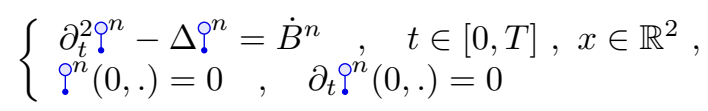

falls within the class of standard hyperbolic systems, for which a unique (global) solution ? $?^{n}$ is known to exist. Then we define the (smooth) approximated path $\Psi^{n} \triangleq\left(?^{n}\right.$, q $\left.^{n}, q^{n}, q \wp^{n}\right)$ along the explicit formulas

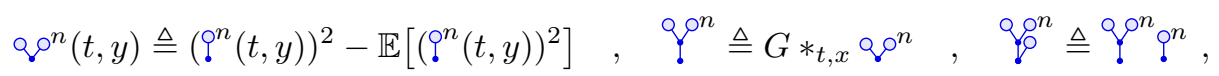

and study the (almost sure) convergence of these processes in suitable spaces.

In order to implement this standard procedure, we will consider here the approximation derived from the so-called harmonizable representation of the space-time fractional Brownian motion, that is the formula (valid for every $H=\left(H_{0}, H_{1}, H_{2}\right) \in(0,1)^{3}$ )

$$
B_{t}\left(x_{1}, x_{2}\right)=c_{H} \int_{\xi \in \mathbb{R}} \int_{\eta \in \mathbb{R}^{2}} \widehat{W}(d \xi, d \eta) \frac{e^{\imath t \xi}-1}{|\xi|^{H_{0}+\frac{1}{2}}} \frac{e^{\imath x_{1} \eta_{1}}-1}{\left|\eta_{1}\right|^{H_{1}+\frac{1}{2}}} \frac{e^{\imath x_{2} \eta_{2}}-1}{\left|\eta_{2}\right|^{H_{2}+\frac{1}{2}}},
$$

where $c_{H}>0$ is a suitable constant and $\widehat{W}$ stands for the Fourier transform of a space-time white noise in $\mathbb{R}^{3}$, defined on some complete filtered probability space $(\Omega, \mathcal{F}, \mathbb{P})$. The approximation $\left(B^{n}\right)_{n \geq 0}$ of $B$ is now defined as $B^{0} \equiv 0$ and, for $n \geq 1$,

$$
B_{t}^{n}\left(x_{1}, x_{2}\right) \triangleq c_{H} \int_{|\xi| \leq 2^{n}} \int_{|\eta| \leq 2^{n}} \widehat{W}(d \xi, d \eta) \frac{e^{\imath t \xi}-1}{|\xi|^{H_{0}+\frac{1}{2}}} \frac{e^{\imath x_{1} \eta_{1}}-1}{\left|\eta_{1}\right|^{H_{1}+\frac{1}{2}}} \frac{e^{\imath x_{2} \eta_{2}}-1}{\left|\eta_{2}\right|^{H_{2}+\frac{1}{2}}}
$$

It is readily checked that for all fixed $H=\left(H_{0}, H_{1}, H_{2}\right) \in(0,1)^{3}$ and $n \geq 1$, the process $B^{n}$ indeed corresponds (almost surely) to a smooth function with respect to all its parameters, and we can thus consider the process $\Psi^{n}$ associated with its derivative $\dot{B}^{n}$ (along (13)-(14)) .

With this notation in mind, our main stochastic result can be stated as follows:

Proposition 1.3. Let $\left(H_{0}, H_{1}, H_{2}\right) \in(0,1)^{3}$ be such that

$$
0<H_{1}<\frac{3}{4} \quad, \quad 0<H_{2}<\frac{3}{4} \quad, \quad 1<H_{0}+H_{1}+H_{2} \leq \frac{5}{4},
$$

Then, for all smooth compactly-supported function $\rho: \mathbb{R}^{2} \rightarrow \mathbb{R}$, all $T>0, p \geq 2$ and

$$
\alpha>\frac{3}{2}-\left(H_{0}+H_{1}+H_{2}\right)
$$

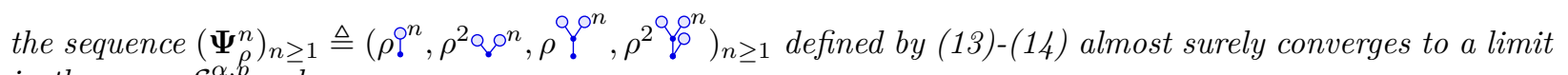
in the space $\mathcal{E}_{T}^{\alpha, p}$, where

$$
\mathcal{E}_{T}^{\alpha, p} \triangleq L_{T}^{\infty} \mathcal{W}^{-\alpha, p} \times L_{T}^{\infty} \mathcal{W}^{-2 \alpha, p} \times L_{T}^{\infty} \mathcal{W}^{1-2 \alpha, p} \times L_{T}^{\infty} \mathcal{W}^{-\alpha, p}
$$

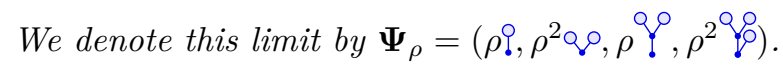


The condition $H_{0}+H_{1}+H_{2}>1$ that appears in (16) turns out to be optimal in the framework of our strategy, as shown by the following divergence property:

Proposition 1.4. Let $\left(H_{0}, H_{1}, H_{2}\right) \in(0,1)^{3}$ be such that

$$
H_{0}+H_{1}+H_{2} \leq 1
$$

and let $\rho: \mathbb{R}^{2} \rightarrow \mathbb{R}$ be a non-zero, smooth and compactly-supported function. Then, for all $\alpha>0$ and $t>0$, one has $\mathbb{E}\left[\left\|\rho^{2} Q \rho^{n}(t, .)\right\|_{\mathcal{W}^{-2 \alpha, 2}\left(\mathbb{R}^{2}\right)}^{2}\right] \rightarrow \infty$ as $n \rightarrow \infty$.

\subsection{Main results}

Let us recall that, for the rest of the paper, we have fixed a smooth function $\rho: \mathbb{R}^{2} \rightarrow \mathbb{R}$ with support included in $D \triangleq\{|x| \leq 2\}$ and satisfying $\rho(x)=1$ for $|x| \leq 1$. Based on the considerations of Section 1.1 and relying on the result of Proposition 1.3, the following definition of a (local-in-space) solution for (1) naturally arises:

Definition 1.5. Fix $\left(H_{0}, H_{1}, H_{2}\right) \in(0,1)^{3}$ such that condition (16) is satisfied, and for every $T>0$, let

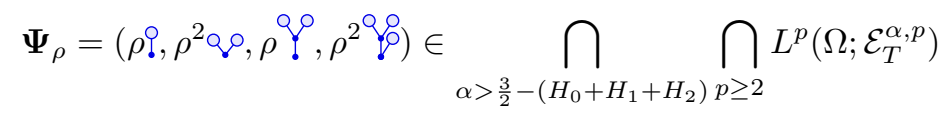

be the process defined through Proposition 1.3. A stochastic process $(u(t, x))_{t \in[0, T], x \in \mathbb{R}^{2}}$ is said to be a Wick solution $($ on $[0, T])$ of the equation

$$
\left\{\begin{array}{l}
\partial_{t}^{2} u-\Delta u=\rho^{2} u^{2}+\dot{B}, \quad x \in \mathbb{R}^{2} \\
u(0, .)=\phi_{0} \quad, \quad \partial_{t} u(0, .)=\phi_{1}
\end{array}\right.
$$

if, almost surely, the auxiliary process $w \triangleq u-?-?$ is a mild solution (on $[0, T]$ ) of the equation

$$
\begin{aligned}
& w=\partial_{t}\left(G_{t} *_{x} \phi_{0}\right)+G_{t} *_{x} \phi_{1} \\
& +G *_{t, x}\left(\rho^{2} w^{2}\right)+G *_{t, x}\left((\rho !)^{2}\right)+2 G *_{t, x}((\rho w) \cdot(\rho !))+2 G *_{t, x}((\rho w) \cdot(\rho !))+2 G *_{t, x}\left(\rho^{2} q_{\ell}:\right) .
\end{aligned}
$$

Our main results regarding equation (20) finally read as follows:

Theorem 1.6. Let $\left(\phi_{0}, \phi_{1}\right) \in \mathcal{W}^{\frac{1}{2}, 2}\left(\mathbb{R}^{2}\right) \times \mathcal{W}^{-\frac{1}{2}, 2}\left(\mathbb{R}^{2}\right)$ and let $\left(H_{0}, H_{1}, H_{2}\right) \in(0,1)^{3}$ be such that

$$
0<H_{1}<\frac{3}{4} \quad, \quad 0<H_{2}<\frac{3}{4} \quad, \quad 1<H_{0}+H_{1}+H_{2} \leq \frac{5}{4} .
$$

Then, almost surely, there exists a time $T_{0}>0$ such that Equation (20) admits a unique Wick solution $u$ in the set

$$
\left\{u \in \mathcal{S}^{\prime}\left(\mathbb{R}^{2}\right): u=?+\varrho^{\circ}+w, \text { with } w \in L_{T_{0}}^{\infty} \mathcal{W}^{\frac{1}{2}, 2}\right\} .
$$

Using the continuity properties of equation (21) with respect to $\boldsymbol{\Psi}$ (see estimate (30) below), we are also able to offer the following sequential approach to the problem: 
Theorem 1.7. Under the assumptions of Theorem 1.6, consider the sequence $\left(u^{n}\right)_{n \geq 0}$ of (classical) solutions to the renormalized equation

$$
\left\{\begin{array}{l}
\partial_{t}^{2} u^{n}-\Delta u^{n}=\rho^{2}\left(u^{n}\right)^{2}-\sigma^{n}+\left(1-\rho^{2}\right)\left(\varphi^{n}\right)^{2}+\dot{B}^{n} \quad, \quad x \in \mathbb{R}^{2}, \\
u^{n}(0, .)=\phi_{0} \quad, \quad \partial_{t} u^{n}(0, .)=\phi_{1},
\end{array}\right.
$$

where $\sigma_{t}^{n}(x) \triangleq \mathbb{E}\left[\left(?_{t}^{n}(x)\right)^{2}\right]$. Then $\sigma_{t}^{n}(x)$ is independent of $x$, one has

$$
\sigma_{t}^{n} \stackrel{n \rightarrow \infty}{\sim} c_{H}^{1} t 2^{2 n\left(\frac{3}{2}-\left(H_{0}+H_{1}+H_{2}\right)\right)}
$$

for some constant $c_{H}^{1}$, and, almost surely, there exists a time $T_{0}>0$ such that the sequence $\left(u^{n}\right)$ converges in the space $L^{\infty}\left(\left[0, T_{0}\right] ; \mathcal{W}^{-\alpha, 2}(\{|x| \leq 1\})\right)$ to the solution u exhibited in Theorem 1.6, for every $\alpha>\frac{3}{2}-$ $\left(H_{0}+H_{1}+H_{2}\right)$.

Theorems 1.6 and 1.7 thus correspond to the third-order extensions of Theorems 1.6 and 1.7 in [1] (which only covered the situation where $H_{0}+H_{1}+H_{2}>\frac{5}{4}$ ), and they allow us to handle the fractional problem up to the critical threshold $H_{0}+H_{1}+H_{2}=1$ (where "criticality" is here understood in the sense of Proposition 1.4, see Remark 1.10 below). To some extent, these statements can be compared with the results of [4, Theorem 1.1 and Theorem 1.12] for the three-dimensional white-noise-driven model.

Remark 1.8. On the unit ball $\{|x| \leq 1\}$, that is on the space-domain we actually focus, equation (23) reduces to the Wick-renormalized model $\partial_{t}^{2} u^{n}-\Delta u^{n}=\left[\left(u^{n}\right)^{2}-\sigma^{n}\right]+\dot{B}^{n}$, as expected. In fact, a global-in-space solution (i.e. the consideration of $\rho \equiv 1$ on $\mathbb{R}^{2}$ in the above results) could perhaps be obtained through the involvement of weighted spaces in the subsequent analysis, both in the convergence results of Proposition 1.3 and in the study of the deterministic equation. Nevertheless, we expect this extension to be the source of technical stability issues (see for instance the developments in [5] for the parabolic setting) and therefore we postpone these investigations to a (possible) future publication. On the other hand, studying the equation on the 2D-torus (with appropriate boundary conditions) should certainly give rise to very similar estimates and results. This would however require the introduction of a fractional Brownian noise on the torus, a model that we find slightly more "exotic" than ours.

Remark 1.9. At the level of the approximated equation (23), another possibility would be to consider, just as in [1, Theorem 1.7], the approximation given by

$$
\partial_{t}^{2} u^{n}-\Delta u^{n}=\rho^{2}\left(\left(u^{n}\right)^{2}-\sigma^{n}\right)+\dot{B}^{n} .
$$

However, expanding equation (25) along the pattern of Section 1.1 readily leads us to the consideration of the alternative path

$$
\widetilde{\Psi}_{\rho}^{n} \triangleq\left(\rho !^{n}, \rho^{2} Q \rho^{n}, \rho\left[G *_{t, x}\left(\rho^{2} Q \rho^{n}\right)\right],\left(\rho\left[G *_{t, x}\left(\rho^{2} Q \rho^{n}\right)\right]\right)\left(\rho !^{n}\right)\right),
$$

which we have found less elegant (and slightly more difficult to handle) than the path

$$
\Psi_{\rho}^{n}=\left(\rho \varphi^{n}, \rho^{2} \otimes \rho^{n}, \rho !^{n}, \rho^{2} \varphi^{n}\right)
$$

at the core of the above analysis (see Section 2.3 for more details on the transition from (23) to (21)).

Remark 1.10. The claimed optimality proved through Proposition 1.4 is of course relative to our specific procedure, which strongly relies on the Wick-renormalized product of ?, and we do not pretend that any other approach to the equation would fail likewise. In fact, we think that our strategy induces two major restrictions in this context.

First, it only allows us to consider the central elements,, 0, ,, ,, as functions of time (with values in some Sobolev spaces). But these stochastic elements could be more generally interpreted as space-time 
distributions in a weak sense, that is when integrated against smooth test-functions $\varphi(t, x)$, and maybe the divergence problem for $\mathrm{Q}^{n}$ could be overcome along this interpretation. However, it is not exactly clear to us how to combine the latter (weak) interpretation with the Strichartz-type controls at the core of the subsequent analysis.

Secondly, Proposition 1.4 is only based on the consideration of the approximated noise (15) and the Wickrenormalization method. Perhaps some more sophisticated approximation of the noise and/or some more sophisticated renormalization procedure would yield a positive result in the rougher situation $H_{0}+H_{1}+H_{2} \leq$ 1.

The rest of the paper is organized along a natural two-part splitting. In Section 2, we will handle the deterministic aspects of the problem, that is the well-posedness of equation (21) once endowed with a path $\Psi_{\rho}=\left(\rho \uparrow, \rho^{2} Q \rho, \rho \uparrow, \rho^{2} q\right) \in \mathcal{E}_{T}^{\alpha, p}$. The proofs of Theorem 1.6 and Theorem 1.7 will almost immediately follow (Section 2.3). Section 3 will then be devoted to the technical stochastic estimates behind the convergence statement of Proposition 1.3 and the explosion phenomenon of Proposition 1.4.

Throughout the paper, we will denote by $A \lesssim B$ any estimate of the form $A \leq c B$, where $c>0$ is a constant that does not depend on the parameters under consideration.

Acknowledgements. I am grateful to Laurent Thomann for his encouragements and for his advice about the treatment of the deterministic equation. I also deeply thank two anonymous reviewers for their careful reading of the paper: their comments and questions have led to significant improvements in the presentation of these results, and they have also allowed me to fix a few mistakes in the first version of the manuscript.

\section{Auxiliary (deterministic) equation}

Let us first recall that for all parameters $\alpha>0, p \geq 1, T>0$, the functional space $\mathcal{E}_{T}^{\alpha, p}$ has been introduced in (18). Also, for the whole study, we have fixed a smooth cut-off functions $\rho$ with support in $\{|x| \leq 2\}$ (such that $\rho(x)=1$ for $|x| \leq 1)$, and for this reason let us set $D \triangleq\{|x| \leq 2\}$ for the rest of the section.

At some point of the analysis, we will need to use the fact that the noise components we are implicitly interested in, that is the distributions $(\rho),\left(\rho^{2} \odot \rho\right),(\rho \varphi),\left(\rho^{2} Q \varnothing\right)$ given by Proposition 1.3, are supported by the compact domain $D$. Therefore, in the sequel, we will consider the subset

$$
\mathcal{E}_{T, D}^{\alpha, p} \triangleq\left\{\boldsymbol{\Psi}=\left(\boldsymbol{\Psi}^{\mathbf{1}}, \mathbf{\Psi}^{\mathbf{2}}, \boldsymbol{\Psi}^{\mathbf{3}}, \boldsymbol{\Psi}^{\mathbf{4}}\right) \in \mathcal{E}_{T}^{\alpha, p}: \operatorname{supp}\left(\boldsymbol{\Psi}^{\mathbf{i}}\right) \subset D \text { for } i=1, \ldots, 4\right\} .
$$

Now, for a fixed element $\boldsymbol{\Psi}=\left(\boldsymbol{\Psi}^{\mathbf{1}}, \mathbf{\Psi}^{\mathbf{2}}, \boldsymbol{\Psi}^{\mathbf{3}}, \boldsymbol{\Psi}^{\mathbf{4}}\right) \in \mathcal{E}_{T, D}^{\alpha, p}$ (with suitable $\left.\alpha, p\right)$ and $\phi_{0}, \phi_{1}$ in appropriate Sobolev spaces, our objective here is to settle a (local in time) fixed-point argument for the mild equation

$$
\begin{aligned}
w= & \partial_{t}\left(G_{t} *_{x} \phi_{0}\right)+G_{t} *_{x} \phi_{1} \\
& +G *_{t, x}\left(\rho^{2} w^{2}\right)+G *_{t, x}\left(\left(\boldsymbol{\Psi}^{\mathbf{3}}\right)^{2}\right)+2 G *_{t, x}\left((\rho w) \cdot \mathbf{\Psi}^{\mathbf{3}}\right)+2 G *_{t, x}\left((\rho w) \cdot \boldsymbol{\Psi}^{\mathbf{1}}\right)+2 G *_{t, x} \mathbf{\Psi}^{\mathbf{4}} .
\end{aligned}
$$

\subsection{Basic preliminary results}

As we mentionned it in the introduction, convolving with the wave kernel is known to entail specific regularization effects, that are generally summed up through the so-called Strichartz inequalities (see [2]). It turns out that the following (much) weaker result will be sufficient for our purpose here: 
Proposition 2.1. (i) For all $0 \leq T \leq 1$ and $w \in L_{T}^{1} \mathcal{W}^{-\frac{1}{2}, 2}$, it holds that

$$
\mathcal{N}\left[G *_{t, x} w ; L_{T}^{\infty} \mathcal{W}^{\frac{1}{2}, 2}\right] \lesssim \mathcal{N}\left[w ; L_{T}^{2} \mathcal{W}^{-\frac{1}{2}, 2}\right] .
$$

(ii) For all $0 \leq T \leq 1$ and $\left(\phi_{0}, \phi_{1}\right) \in \mathcal{W}^{\frac{1}{2}, 2}\left(\mathbb{R}^{2}\right) \times \mathcal{W}^{-\frac{1}{2}, 2}\left(\mathbb{R}^{2}\right)$, it holds that

$$
\mathcal{N}\left[\partial_{t}\left(G_{t} *_{x} \phi_{0}\right) ; L_{T}^{\infty} \mathcal{W}^{\frac{1}{2}, 2}\right] \lesssim\left\|\phi_{0}\right\|_{\mathcal{W}^{\frac{1}{2}, 2}\left(\mathbb{R}^{2}\right)} \quad \text { and } \quad \mathcal{N}\left[G_{t} *_{x} \phi_{1} ; L_{T}^{\infty} \mathcal{W}^{\frac{1}{2}, 2}\right] \lesssim\left\|\phi_{1}\right\|_{\mathcal{W}^{-\frac{1}{2}, 2}\left(\mathbb{R}^{2}\right)} .
$$

Proof. Both (27) and (28) rely on elementary estimates only.

(i) Observe first that

$$
\mathcal{F}_{x}\left(\left[G *_{t, x} w\right](t, .)\right)(\xi)=\int_{0}^{t} d s\left(\mathcal{F}_{x} G_{t-s}\right)(\xi)\left(\mathcal{F}_{x} w_{s}\right)(\xi),
$$

so that, for every $t \in[0, T]$,

$$
\begin{aligned}
\int_{\mathbb{R}^{2}} d \xi\left\{1+|\xi|^{2}\right\}^{\frac{1}{2}}\left|\mathcal{F}_{x}\left(\left[G *_{t, x} w\right](t, .)\right)(\xi)\right|^{2} & \leq \int_{0}^{t} d s \int_{\mathbb{R}^{2}} d \xi\left\{1+|\xi|^{2}\right\}^{\frac{1}{2}} \frac{\sin ^{2}((t-s)|\xi|)}{|\xi|^{2}}\left|\left(\mathcal{F}_{x} w_{s}\right)(\xi)\right|^{2} \\
& \lesssim \int_{0}^{t} d s \int_{\mathbb{R}^{2}} d \xi\left\{1+|\xi|^{2}\right\}^{-\frac{1}{2}}\left|\left(\mathcal{F}_{x} w_{s}\right)(\xi)\right|^{2}
\end{aligned}
$$

hence the conclusion.

(ii) The bound for $\mathcal{N}\left[G_{t} *_{x} \phi_{1} ; L_{T}^{\infty} \mathcal{W}^{\frac{1}{2}, 2}\right]$ follows from similar arguments as above. Then, along the same idea, observe that

$$
\mathcal{F}_{x}\left(\partial_{t}\left(G . *_{x} \phi_{0}\right)(t, .)\right)(\xi)=\partial_{t}\left(\mathcal{F}_{x} G\right)_{t}(\xi)\left(\mathcal{F}_{x} \phi_{0}\right)(\xi)=\cos (t|\xi|)\left(\mathcal{F}_{x} \phi_{0}\right)(\xi),
$$

and so, for every $t \in[0, T]$,

$$
\int_{\mathbb{R}^{2}} d \xi\left\{1+|\xi|^{2}\right\}^{\frac{1}{2}}\left|\mathcal{F}_{x}\left(\partial_{t}\left(G . *_{x} \phi_{0}\right)(t, .)\right)(\xi)\right|^{2} \leq \int_{\mathbb{R}^{2}} d \xi\left\{1+|\xi|^{2}\right\}^{\frac{1}{2}}\left|\left(\mathcal{F}_{x} \phi_{0}\right)(\xi)\right|^{2} .
$$

Then, in order to control the product operations involved in (26), we will appeal to the following standard properties, the proof of which can for instance be found in [8, Chapter 4]:

Proposition 2.2. (i) For all $\alpha, \beta>0$ and $0<p, p_{1}, p_{2} \leq \infty$ such that

$$
\frac{1}{p} \leq \frac{1}{p_{1}}+\frac{1}{p_{2}} \quad, \quad 0<\alpha<\beta<\frac{2}{p_{2}} \quad, \quad \min \left(\frac{2}{p}+\alpha, 2\right)>\left(\frac{2}{p_{1}}+\alpha\right)+\left(\frac{2}{p_{2}}-\beta\right),
$$

one has

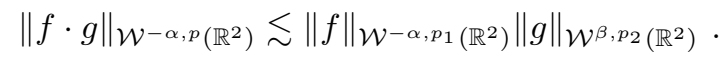

(ii) For all $\beta>\frac{1}{2}, 0<\alpha<\beta$ and $p>2$, one has

$$
\|f \cdot g\|_{\mathcal{W}^{-\alpha, p}\left(\mathbb{R}^{2}\right)} \lesssim\|f\|_{\mathcal{W}^{-\alpha, p}\left(\mathbb{R}^{2}\right)}\|g\|_{\mathcal{W}^{\beta, 2}\left(\mathbb{R}^{2}\right)} .
$$

(iii) For all $\alpha>0$ and $1 \leq p, p_{1}, p_{2} \leq \infty$ such that

$$
\frac{1}{p_{1}}+\frac{1}{p_{2}}=\frac{1}{p} \quad \text { and } \quad 0<\alpha<\min \left(\frac{2}{p_{1}}, \frac{2}{p_{2}}\right),
$$

one has

$$
\|f \cdot g\|_{\mathcal{W}^{\alpha, p}\left(\mathbb{R}^{2}\right)} \lesssim\|f\|_{\mathcal{W}^{\alpha, p_{1}}\left(\mathbb{R}^{2}\right)}\|g\|_{\mathcal{W}^{\alpha, p_{2}\left(\mathbb{R}^{2}\right)}}
$$




\subsection{Solving the deterministic equation}

We are now in a position to exhibit suitable bounds for (26).

Proposition 2.3. Fix $\alpha \in\left(\frac{1}{4}, \frac{1}{2}\right)$ and for all $T>0, \phi=\left(\phi_{0}, \phi_{1}\right) \in \mathcal{W}^{\frac{1}{2}, 2}\left(\mathbb{R}^{2}\right) \times \mathcal{W}^{-\frac{1}{2}, 2}\left(\mathbb{R}^{2}\right)$ and $\boldsymbol{\Psi}=\left(\boldsymbol{\Psi}^{\mathbf{1}}, \mathbf{\Psi}^{\mathbf{2}}, \mathbf{\Psi}^{\mathbf{3}}, \mathbf{\Psi}^{\mathbf{4}}\right) \in \mathcal{E}_{T, D}^{\alpha, 4}$, consider the map $\Gamma_{T, \phi, \boldsymbol{\Psi}}: L_{T}^{\infty} \mathcal{W}^{\frac{1}{2}, 2} \rightarrow L_{T}^{\infty} \mathcal{W}^{\frac{1}{2}, 2}$ given by

$$
\begin{aligned}
& \Gamma_{T, \phi, \Psi}(w) \triangleq \partial_{t}\left(G_{t} *_{x} \phi_{0}\right)+G_{t} *_{x} \phi_{1} \\
& \quad+G *_{t, x}\left(\rho^{2} w^{2}\right)+G *_{t, x}\left(\left(\boldsymbol{\Psi}^{\mathbf{3}}\right)^{2}\right)+2 G *_{t, x}\left((\rho w) \cdot \boldsymbol{\Psi}^{\mathbf{3}}\right)+2 G *_{t, x}\left((\rho w) \cdot \mathbf{\Psi}^{\mathbf{1}}\right)+2 G *_{t, x} \mathbf{\Psi}^{\mathbf{4}} .
\end{aligned}
$$

Then, for all $T>0, \phi=\left(\phi_{0}, \phi_{1}\right) \in \mathcal{W}^{\frac{1}{2}, 2}\left(\mathbb{R}^{2}\right) \times \mathcal{W}^{-\frac{1}{2}, 2}\left(\mathbb{R}^{2}\right), \mathbf{\Psi}_{1}, \mathbf{\Psi}_{2} \in \mathcal{E}_{T, D}^{\alpha, 4}$ and $w, w_{1}, w_{2} \in L_{T}^{\infty} \mathcal{W}^{\frac{1}{2}, 2}$, the following bounds hold true:

$$
\begin{aligned}
& \mathcal{N}\left[\Gamma_{T, \phi, \Psi_{1}}(w) ; L_{T}^{\infty} \mathcal{W}^{\frac{1}{2}, 2}\right] \lesssim\left\|\phi_{0}\right\|_{\mathcal{W}^{\frac{1}{2}, 2}}+\left\|\phi_{1}\right\|_{\mathcal{W}^{-\frac{1}{2}, 2}} \\
&+T^{1 / 2}\left\{\mathcal{N}\left[w ; L_{T}^{\infty} \mathcal{W}^{\frac{1}{2}, 2}\right]^{2}+\left\|\boldsymbol{\Psi}_{1}\right\|^{2}+\left\|\boldsymbol{\Psi}_{1}\right\| \mathcal{N}\left[w ; L_{T}^{\infty} \mathcal{W}^{\frac{1}{2}, 2}\right]+\left\|\boldsymbol{\Psi}_{1}\right\|\right\}
\end{aligned}
$$

and

$$
\begin{aligned}
& \mathcal{N}\left[\Gamma_{T, \phi, \boldsymbol{\Psi}_{1}}\left(w_{1}\right)-\Gamma_{T, \phi, \mathbf{\Psi}_{2}}\left(w_{2}\right) ; L_{T}^{\infty} \mathcal{W}^{\frac{1}{2}, 2}\right] \\
& \qquad T^{1 / 2}\left[\mathcal{N}\left[w_{1}-w_{2} ; L_{T}^{\infty} \mathcal{W}^{\frac{1}{2}, 2}\right]\left\{\mathcal{N}\left[w_{1} ; L_{T}^{\infty} \mathcal{W}^{\frac{1}{2}, 2}\right]+\mathcal{N}\left[w_{2} ; L_{T}^{\infty} \mathcal{W}^{\frac{1}{2}, 2}\right]\right\}+\left\|\boldsymbol{\Psi}_{1}-\mathbf{\Psi}_{2}\right\|\left\{\left\|\boldsymbol{\Psi}_{1}\right\|+\left\|\boldsymbol{\Psi}_{2}\right\|\right\}\right. \\
& \left.\quad+\left\|\boldsymbol{\Psi}_{1}-\mathbf{\Psi}_{2}\right\| \mathcal{N}\left[w_{1} ; L_{T}^{\infty} \mathcal{W}^{\frac{1}{2}, 2}\right]+\left\|\boldsymbol{\Psi}_{2}\right\| \mathcal{N}\left[w_{1}-w_{2} ; L_{T}^{\infty} \mathcal{W}^{\frac{1}{2}, 2}\right]+\left\|\boldsymbol{\Psi}_{1}-\boldsymbol{\Psi}_{2}\right\|\right]
\end{aligned}
$$

where the proportional constants only depend on $\alpha$, and the norm $\|\cdot\|$ is naturally defined as

$$
\begin{aligned}
& \|\boldsymbol{\Psi}\|=\|\boldsymbol{\Psi}\|_{\mathcal{E}_{T}^{\alpha, 4}} \\
& \triangleq \mathcal{N}\left[\Psi^{\mathbf{1}} ; L_{T}^{\infty} \mathcal{W}^{-\alpha, 4}\right]+\mathcal{N}\left[\Psi^{\mathbf{2}} ; L_{T}^{\infty} \mathcal{W}^{-2 \alpha, 4}\right]+\mathcal{N}\left[\boldsymbol{\Psi}^{\mathbf{3}} ; L_{T}^{\infty} \mathcal{W}^{1-2 \alpha, 4}\right]+\mathcal{N}\left[\Psi^{\mathbf{4}} ; L_{T}^{\infty} \mathcal{W}^{-\alpha, 4}\right] .
\end{aligned}
$$

Proof. As expected, the two bounds (29) and (30) will follow from the combination of the estimates in Proposition 2.1 and Proposition 2.2. The elementary Sobolev embedding

$$
\mathcal{W}^{s_{0}, p_{0}}(D) \subset \mathcal{W}^{s_{1}, p_{1}}(D) \quad \text { if } s_{0}-\frac{2}{p_{0}} \geq s_{1}-\frac{2}{p_{1}}
$$

will also be requested. For the sake of clarity, let us set $\mathcal{W}_{D}^{s, p} \triangleq \mathcal{W}^{s, p}(D)$ and $L_{D}^{p} \triangleq \mathcal{W}^{0, p}(D)$.

Initial conditions: the bound for $\mathcal{N}\left[\partial_{t}\left(G_{t} *_{x} \phi_{0}\right)+G_{t} *_{x} \phi_{1} ; L_{T}^{\infty} \mathcal{W}^{\frac{1}{2}, 2}\right]$ immediately follows from Proposition 2.1 , item (ii).

Bound on $G *_{t, x}\left(\rho^{2} w^{2}\right)$ : Using successively (27) and (31), we deduce that

$$
\begin{aligned}
& \mathcal{N}\left[G *_{t, x}\left(\rho^{2} w^{2}\right) ; L_{T}^{\infty} \mathcal{W}^{\frac{1}{2}, 2}\right] \\
& \lesssim \mathcal{N}\left[\rho^{2} w^{2} ; L_{T}^{2} \mathcal{W}_{D}^{-\frac{1}{2}, 2}\right] \lesssim \mathcal{N}\left[\rho^{2} w^{2} ; L_{T}^{2} L_{D}^{\frac{4}{3}}\right] \lesssim \mathcal{N}\left[w ; L_{T}^{4} L_{D}^{\frac{8}{3}}\right]^{2} \lesssim T^{1 / 2} \mathcal{N}\left[w ; L_{T}^{\infty} L_{D}^{\frac{8}{3}}\right]^{2}
\end{aligned}
$$

and we get the expected bound through the embedding $\mathcal{W}_{D}^{\frac{1}{2}, 2} \subset L_{D}^{\frac{8}{3}}$.

Bound on $G *_{t, x}\left(\left(\Psi_{1}^{3}\right)^{2}\right)$ : Just as above, we have

$$
\mathcal{N}\left[G *_{t, x}\left(\left(\boldsymbol{\Psi}_{1}^{\mathbf{3}}\right)^{2}\right) ; L_{T}^{\infty} \mathcal{W}^{\frac{1}{2}, 2}\right] \lesssim T^{1 / 2} \mathcal{N}\left[\mathbf{\Psi}_{1}^{\mathbf{3}} ; L_{T}^{\infty} L_{D}^{\frac{8}{3}}\right]^{2},
$$


and the desired bound is here obtained through the embedding $\mathcal{W}_{D}^{1-2 \alpha, 4} \subset L_{D}^{\frac{8}{3}}$.

Bound on $G *_{t, x}\left((\rho w) \cdot \mathbf{\Psi}_{1}^{3}\right)$ : Let $1<\tilde{r}_{1}<2$ be defined by the relation

$$
\frac{1}{\tilde{r}_{1}}=\frac{5}{4}-\alpha
$$

Using successively (27) and (31), we get that

$$
\mathcal{N}\left[G *_{t, x}\left((\rho w) \cdot \mathbf{\Psi}_{1}^{\mathbf{3}} ; L_{T}^{\infty} \mathcal{W}^{\frac{1}{2}, 2}\right] \lesssim \mathcal{N}\left[(\rho w) \cdot \boldsymbol{\Psi}_{1}^{\mathbf{3}} ; L_{T}^{2} \mathcal{W}_{D}^{-\frac{1}{2}, 2}\right] \lesssim T^{1 / 2} \mathcal{N}\left[(\rho w) \cdot \boldsymbol{\Psi}_{1}^{\mathbf{3}} ; L_{T}^{\infty} \mathcal{W}_{D}^{1-2 \alpha, \tilde{r}_{1}}\right]\right.
$$

Then introduce the additional parameter $2 \leq p_{1} \leq 4$ defined by

$$
\frac{1}{p_{1}}=\frac{1}{\tilde{r}_{1}}-\frac{1}{2}=\frac{3}{4}-\alpha .
$$

By Proposition 2.2 (item (iii)), we know that for each fixed $t \geq 0$,

$$
\begin{aligned}
& \left\|(\rho w)_{t} \cdot\left(\mathbf{\Psi}_{1}^{\mathbf{3}}\right)_{t}\right\|_{\mathcal{W}^{1-2 \alpha, \tilde{r}_{1}\left(\mathbb{R}^{2}\right)}} \lesssim\left\|(\rho w)_{t}\right\|_{\mathcal{W}^{1-2 \alpha, 2}\left(\mathbb{R}^{2}\right)}\left\|\left(\mathbf{\Psi}_{1}^{\mathbf{3}}\right)_{t}\right\|_{\mathcal{W}^{1-2 \alpha, p_{1}\left(\mathbb{R}^{2}\right)}} \\
& \lesssim\left\|w_{t}\right\|_{\mathcal{W}_{D}^{\frac{1}{2}, 2}}\left\|\left(\mathbf{\Psi}_{1}^{\mathbf{3}}\right)_{t}\right\|_{\mathcal{W}_{D}^{1-2 \alpha, 4}}
\end{aligned}
$$

which immediately yields

$$
\begin{aligned}
& \mathcal{N}\left[G *_{t, x}\left((\rho w) \cdot \boldsymbol{\Psi}_{1}^{3}\right) ; L_{T}^{\infty} \mathcal{W}^{\frac{1}{2}, 2}\right] \\
& \lesssim T^{1 / 2} \mathcal{N}\left[w ; L_{T}^{\infty} \mathcal{W}^{\frac{1}{2}, 2}\right] \mathcal{N}\left[\mathbf{\Psi}_{1}^{3} ; L_{T}^{\infty} \mathcal{W}^{1-2 \alpha, 4}\right] \lesssim T^{1 / 2} \mathcal{N}\left[w ; L_{T}^{\infty} \mathcal{W}^{\frac{1}{2}, 2}\right]\left\|\Psi_{1}\right\|
\end{aligned}
$$

Bound on $G *_{t, x}\left((\rho w) \cdot \boldsymbol{\Psi}_{1}^{\mathbf{1}}\right)$ : Let $1<\tilde{r}_{2}<2$ be defined by the relation

$$
\frac{1}{\tilde{r}_{2}}=\frac{3}{4}-\frac{\alpha}{2}
$$

Using successively (27) and (31), we get that

$$
\mathcal{N}\left[G *_{t, x}\left((\rho w) \cdot \boldsymbol{\Psi}_{1}^{\mathbf{1}}\right) ; L_{T}^{\infty} \mathcal{W}^{\frac{1}{2}, 2}\right] \lesssim \mathcal{N}\left[(\rho w) \cdot \boldsymbol{\Psi}_{1}^{\mathbf{1}} ; L_{T}^{2} \mathcal{W}_{D}^{-\frac{1}{2}, 2}\right] \lesssim T^{1 / 2} \mathcal{N}\left[(\rho w) \cdot \Psi_{1}^{1} ; L_{T}^{\infty} \mathcal{W}_{D}^{-\alpha, \tilde{r}_{2}}\right] .
$$

Then, using Proposition 2.2 (item $(i)$ and $(i i)$ ), we can assert that for each fixed $t \geq 0$,

$$
\left\|(\rho w)_{t} \cdot\left(\mathbf{\Psi}_{1}^{\mathbf{1}}\right)_{t}\right\|_{\mathcal{W}_{D}^{-\alpha, \tilde{r}_{2}}} \lesssim\left\|(\rho w)_{t}\right\|_{\mathcal{W}_{D}^{\frac{1}{2}, 2}}\left\|\left(\mathbf{\Psi}_{1}^{\mathbf{1}}\right)_{t}\right\|_{\mathcal{W}_{D}^{-\alpha, 4}} \lesssim\left\|w_{t}\right\|_{\mathcal{W}_{D}^{\frac{1}{2}, 2}}\left\|\left(\mathbf{\Psi}_{1}^{\mathbf{1}}\right)_{t}\right\|_{\mathcal{W}^{-\alpha, 4}}
$$

and accordingly

$$
\mathcal{N}\left[G *_{t, x}\left((\rho w) \cdot \mathbf{\Psi}_{1}^{\mathbf{1}}\right) ; L_{T}^{\infty} \mathcal{W}^{\frac{1}{2}, 2}\right] \lesssim T^{1 / 2} \mathcal{N}\left[w ; L_{T}^{\infty} \mathcal{W}^{\frac{1}{2}, 2}\right]\left\|\mathbf{\Psi}_{1}\right\|
$$

Bound on $G *_{t, x} \Psi_{1}^{4}$ : By $(27)$,

$$
\mathcal{N}\left[G *_{t, x} \mathbf{\Psi}_{1}^{4} ; L_{T}^{\infty} \mathcal{W}^{\frac{1}{2}, 2}\right] \lesssim \mathcal{N}\left[\mathbf{\Psi}_{1}^{4} ; L_{T}^{2} \mathcal{W}_{D}^{-\frac{1}{2}, 2}\right] \lesssim T^{1 / 2} \mathcal{N}\left[\mathbf{\Psi}_{1}^{4} ; L_{T}^{\infty} \mathcal{W}_{D}^{-\alpha, 4}\right] \lesssim T^{1 / 2}\left\|\boldsymbol{\Psi}_{1}\right\|
$$

which concludes the proof of (29).

We can then show (30) along similar arguments. 


\subsection{Proof of the main results}

Proof of Theorem 1.6. The combination of (29) and (30) easily allows us to assert that for $T_{0}>0$ small enough and for all $\boldsymbol{\Psi} \in \mathcal{E}_{T_{0}, D}^{\alpha, 4}$ (with $\alpha \in\left(\frac{1}{4}, \frac{1}{2}\right)$ ), the map $\Gamma_{T_{0}, \phi, \Psi}$ is a contraction on some stable subset of $L_{T_{0}}^{\infty} \mathcal{W}_{D}^{\frac{1}{2}, 2}$, which yields a unique solution $w$ for equation (26). Then it suffices of course to apply this result (in an almost sure way) to the stochastic process $\Psi_{\rho}=\left(\rho, \rho^{2} Q_{\rho}, \rho \rho^{\rho}, \rho^{2} \varphi^{Q}\right) \in \mathcal{E}_{T_{0}, D}^{\alpha, 4}$ given by Proposition 1.3 , where $\alpha$ is picked such that $\frac{1}{4} \leq \frac{3}{2}-\left(H_{0}+H_{1}+H_{2}\right)<\alpha<\frac{1}{2}$, and set $u \triangleq ?+$ q $+w$.

Proof of Theorem 1.7. For fixed $n \geq 1$, let $u^{n}$ be the solution of (23) and set $w^{n} \triangleq u^{n}-\varrho^{n}-q^{0}$, where $?^{n}$ and $?^{n}$ are defined in (14). Following the lines of Section 1.1, we can explicitly verify that $w^{n}$ is the solution of (26) associated with the process $\left(\Psi_{\rho}^{n}\right)_{n \geq 1} \triangleq\left(\rho !^{n}, \rho^{2} Q_{\rho} \rho^{n}, \rho \varphi^{\rho^{n}}, \rho^{2} \varphi^{\rho^{n}}\right)$. Observe indeed that the following simplification occurs: setting $v^{n} \triangleq u^{n}-?^{n}$, we have

$$
\begin{aligned}
v^{n} & =\partial_{t}\left(G_{t} *_{x} \phi_{0}\right)+G_{t} *_{x} \phi_{1}+G *_{t, x}\left[\rho^{2}\left(v^{n}+\varphi^{n}\right)^{2}-\sigma^{n}+\left(1-\rho^{2}\right)\left(\varphi^{n}\right)^{2}\right] \\
& =\partial_{t}\left(G_{t} *_{x} \phi_{0}\right)+G_{t} *_{x} \phi_{1}+G *_{t, x}\left[\rho^{2}\left(v^{n}\right)^{2}+2\left(\rho v^{n}\right) \cdot\left(\rho \varphi^{n}\right)\right]+G *_{t, x} Q^{n},
\end{aligned}
$$

and from there it is readily checked that $w^{n}\left(=v^{n}-G *_{t, x}, 0^{n}\right)$ satisfies the expected equation (26). Then, based on (29)-(30), and keeping the result of Proposition 1.3 in mind, the almost sure convergence of $w^{n}$ in $L_{T_{0}}^{\infty} \mathcal{W}^{\frac{1}{2}, 2}$ (for some $T_{0}>0$ ) can be shown with the very same arguments as those of the proof of [1, Theorem 1.7]. The (almost sure) convergence of $\rho u^{n}$ in $L_{T_{0}}^{\infty} \mathcal{W}^{-\alpha, 2}$ immediately follows, which entails the desired (almost sure) convergence of $u^{n}$ in $L_{T_{0}}^{\infty}\left(\mathcal{W}^{-\alpha, 2}(\{|x| \leq 1\})\right)$.

For the asymptotic estimate of $\sigma^{n}$, let us slightly anticipate the notations of Section 3: in particular, using the forthcoming formula (35), we get that

$$
\begin{aligned}
\sigma_{t}^{n}(x)=\mathbb{E}\left[\left(\varrho_{t}^{n}(x)\right)^{2}\right] & =c \int_{|\xi| \leq 2^{n}} \frac{d \xi}{|\xi|^{2 H_{0}-1}} \int_{|\eta| \leq 2^{n}} \frac{d \eta_{1} d \eta_{2}}{\left|\eta_{1}\right|^{2 H_{1}-1}\left|\eta_{2}\right|^{2 H_{2}-1}}\left|\gamma_{t}(\xi,|\eta|)\right|^{2} \\
& =c \int_{0}^{2^{n}} \frac{d r}{r^{2\left(H_{1}+H_{2}\right)-3}} \int_{|\xi| \leq 2^{n}} \frac{d \xi}{|\xi|^{2 H_{0}-1}}\left|\gamma_{t}(\xi, r)\right|^{2}
\end{aligned}
$$

Assertion (24) is now a straightforward consequence of [1, Proposition 2.4].

\section{Stochastic constructions}

Let us now turn to the main technical part of our analysis, namely the proofs of Propositions 1.3 and 1.4, which includes in particular the construction of the central path $\Psi_{\rho}=\left(\rho \circ, \rho^{2} Q \rho, \rho \varphi, \rho^{2} \&: \%\right)$ above the fractional noise. To this end, our arguments will occasionally appeal to some of the technical results of [1]. However, recall that, in comparison with the setting of [1], we are dealing with a rougher situation here and third-order processes must also come into the picture, so that new (sophisticated) estimates shall be required.

Let us start with the introduction of a few convenient notations (related to the wave kernel and the fractional noise) that we will extensively use in the sequel. First, we set, for all $H=\left(H_{0}, H_{1}, H_{2}\right) \in(0,1)^{3}$, $\xi \in \mathbb{R}, \eta \in \mathbb{R}^{2}$ and $r, t \geq 0$,

$$
\begin{aligned}
& \gamma_{t}(\xi, r) \triangleq e^{\imath \xi t} \int_{0}^{t} d s e^{-\imath \xi s} \frac{\sin (s r)}{r} \quad, \quad \gamma_{s, t}(\xi, r) \triangleq \gamma_{t}(\xi, r)-\gamma_{s}(\xi, r), \\
& K^{H}(\eta) \triangleq \frac{\left|\eta_{1}\right|^{1-2 H_{1}}\left|\eta_{2}\right|^{1-2 H_{2}}}{1+|\eta|^{1+2 H_{0}}} .
\end{aligned}
$$


For all $\tau \in\{9,9,9$, ९, ९ $\}, 1 \leq n \leq m$ and $0 \leq s, t \leq 1$, let us also set $\tau_{t}^{n, m} \triangleq \tau_{t}^{m}-\tau_{t}^{n}$, and then, for $f \in\left\{\tau^{n}, \tau^{m}, \tau^{n, m}\right\}, f_{s, t} \triangleq f_{t}-f_{s}$.

With this notation in mind, the following "covariance" identity clearly holds true: for all $a=\left(a_{1}, a_{2}\right)$, resp. $b=\left(b_{1}, b_{2}\right)$, with $a_{i} \in\{n, m,\{n, m\}\}$, resp. $b_{i} \in\{s, t,\{s, t\}\}$, and all $y, \tilde{y} \in \mathbb{R}^{2}$,

$$
\mathbb{E}\left[a_{b_{1}}^{a_{1}}(y) \overline{?_{b_{2}}^{a_{2}}(\tilde{y})}\right]=c_{H} \int_{\mathbb{R}^{2}} d \eta e^{\imath\langle\eta, y\rangle} e^{-\imath\langle\eta, \tilde{y}\rangle} L_{b}^{H, a}(\eta)
$$

where

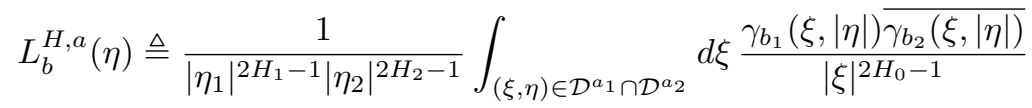

with

$$
\mathcal{D}^{n} \triangleq\left\{|\xi| \leq 2^{n},|\eta| \leq 2^{n}\right\} \quad, \quad \mathcal{D}^{m} \triangleq\left\{|\xi| \leq 2^{m},|\eta| \leq 2^{m}\right\} \quad \text { and } \quad \mathcal{D}^{n, m} \triangleq \mathcal{D}^{m} \backslash \mathcal{D}^{n} .
$$

In the same way, it holds that

$$
\mathbb{E}\left[\varrho_{b_{1}}^{a_{1}}(y) ?_{b_{2}}^{a_{2}}(\tilde{y})\right]=c_{H} \int_{\mathbb{R}^{2}} d \eta e^{\imath\langle\eta, y\rangle} e^{\imath\langle\eta, \tilde{y}\rangle} \tilde{L}_{b}^{H, a}(\eta)
$$

with

$$
\tilde{L}_{b}^{H, a}(\eta) \triangleq \frac{1}{\left|\eta_{1}\right|^{2 H_{1}-1}\left|\eta_{2}\right|^{2 H_{2}-1}} \int_{(\xi, \eta) \in \mathcal{D}^{a_{1}} \cap \mathcal{D}^{a_{2}}} d \xi \frac{\gamma_{b_{1}}(\xi,|\eta|) \gamma_{b_{2}}(\xi,|\eta|)}{|\xi|^{2 H_{0}-1}}
$$

Our estimates toward Proposition 1.3 and Proposition 1.4 will heavily rely on the following bounds for $L_{b}^{H, a}$ :

Lemma 3.1. For all $H=\left(H_{0}, H_{1}, H_{2}\right) \in(0,1)^{3}, \varepsilon \in\left(0, \min \left(H_{0}, \frac{1}{4}\right)\right), 0 \leq n \leq m, 0 \leq s, t, u \leq 1$ and $\eta \in \mathbb{R}^{2}$, it holds that

$$
\max \left(\left|L_{t, t}^{H,(m, m)}(\eta)\right|,\left|\tilde{L}_{t, t}^{H,(m, m)}(\eta)\right|\right) \lesssim K^{H_{\varepsilon, 0}}(\eta)
$$

and

$$
\max \left(\left|L_{(s, t), t}^{H,((n, m), m)}(\eta)\right|,\left|\tilde{L}_{(s, t), t}^{H,((n, m), m)}(\eta)\right|\right) \lesssim 2^{-n \varepsilon}|t-s|^{\varepsilon}\left\{K^{H_{\varepsilon, 0}}(\eta)+K^{H_{\varepsilon, 1}}(\eta)+K^{H_{\varepsilon, 2}}(\eta)\right\},
$$

where $H_{\varepsilon, 0} \triangleq\left(H_{0}-\varepsilon, H_{1}, H_{2}\right), H_{\varepsilon, 1} \triangleq\left(H_{0}, H_{1}-\varepsilon, H_{2}\right), H_{\varepsilon, 2} \triangleq\left(H_{0}, H_{1}, H_{2}-\varepsilon\right)$, and the proportional constants do no depend on $(n, m),(s, t)$ and $\eta$.

Proof. Thanks to [1, Corollary 2.2], we can assert that, under the assumptions of the lemma, and for all $r \geq 0$, one has

$$
\int_{\mathbb{R}} d \xi \frac{\left|\gamma_{s, t}(\xi, r)\right|\left|\gamma_{u}(\xi, r)\right|}{|\xi|^{2 H_{0}-1}} \lesssim \frac{|t-s|^{\varepsilon}}{1+r^{1+2\left(H_{0}-\varepsilon\right)}}
$$

where the proportional constant only depends on $H_{0}$ and $\varepsilon$. Both estimates (36) and (37) immediately follow. 


\subsection{Proof of Proposition 1.3}

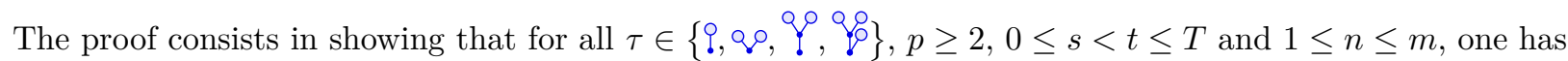

$$
\int_{\mathbb{R}^{2}} d x \mathbb{E}\left[\left|\mathcal{F}_{x}^{-1}\left(\left\{1+|.|^{2}\right\}^{\frac{|\tau|}{2}} \mathcal{F}_{x}\left(\rho \cdot \tau_{s, t}^{n, m}\right)\right)(x)\right|^{2}\right]^{p} \leq c 2^{-2 n \varepsilon p}|t-s|^{2 \varepsilon p},
$$

for some $\varepsilon>0$, some constant $c>0$ that does not depend on $n, m, s, t$, and where the "order" $|\tau|$ of $\tau$ is

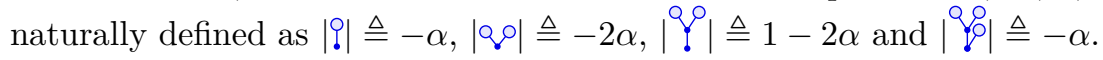

Indeed, once endowed with (39), we can first use the hypercontractivity property of Gaussian chaoses to assert that

$$
\mathbb{E}\left[\mathcal{N}\left[\rho \cdot \tau_{s, t}^{n, m} ; \mathcal{W}^{|\tau|, 2 p}\right]^{2 p}\right] \leq c 2^{-2 n \varepsilon p}|t-s|^{2 \varepsilon p} .
$$

Then, thanks to the Garsia-Rodemich-Rumsey inequality, we get that, for $0<\varepsilon_{0}<\varepsilon$ and for $p$ large enough,

$$
\mathbb{E}\left[\mathcal{N}\left[\rho \cdot \tau^{n, m} ; \mathcal{C}^{\varepsilon_{0}}\left([0, T] ; \mathcal{W}^{|\tau|, 2 p}\right)\right]^{2 p}\right] \leq c 2^{-2 n \varepsilon p},
$$

which ensures the convergence of $\left(\rho \cdot \tau^{n}\right)$ in the space $L^{2 p}\left(\Omega ; \mathcal{C}^{\varepsilon_{0}}\left([0, T] ; \mathcal{W}^{|\tau|, 2 p}\right)\right)$. The desired almost sure convergence finally follows from an application of Borel-Cantelli lemma.

Before we turn to the proof of (39), let us state an elementary technical property that somehow sums up the role of the cut-off function $\rho$ in the subsequent computations:

Lemma 3.2. Fix $\sigma \in \mathbb{R}$ and let $\mathcal{R}: \mathbb{R}^{2} \rightarrow \mathbb{R}$ be defined as

$$
\mathcal{R}(x) \triangleq \iint_{\left(\mathbb{R}^{2}\right)^{2}} \frac{d \lambda d \tilde{\lambda}}{\left\{1+|\lambda|^{2}\right\} \sigma\left\{1+|\tilde{\lambda}|^{2}\right\}^{\sigma}} e^{\imath\langle x, \lambda-\tilde{\lambda}\rangle} \iint_{\left(\mathbb{R}^{2}\right)^{2}} d \xi d \tilde{\xi} \widehat{\rho}(\lambda-\xi) \widehat{\rho}(-\tilde{\lambda}+\tilde{\xi}) \mathcal{Q}(\xi, \tilde{\xi}),
$$

for some integrable function $\mathcal{Q}:\left(\mathbb{R}^{2}\right)^{2} \rightarrow \mathbb{R}$. Then, for every $p \geq 1$, it holds that

$$
\left|\int_{\mathbb{R}^{2}} d x \mathcal{R}(x)^{p}\right| \lesssim\left(\iint_{\left(\mathbb{R}^{2}\right)^{2}} \frac{d \lambda d \tilde{\lambda}}{\left\{1+|\lambda|^{2}\right\}^{\sigma}\left\{1+|\tilde{\lambda}|^{2}\right\}^{\sigma}}|\mathcal{Q}(\lambda, \tilde{\lambda})|\right)^{p},
$$

where the proportional constant depends on $\rho, p$ and $\sigma$.

Proof. One has

$$
\begin{aligned}
& \int_{\mathbb{R}^{2}} d x \mathcal{R}(x)^{p} \\
& =\int_{\mathbb{R}^{2}} d x \prod_{j=1}^{p} \iint_{\left(\mathbb{R}^{2}\right)^{2}} \frac{d \lambda_{j} d \tilde{\lambda}_{j}}{\left\{1+\left|\lambda_{j}\right|^{2}\right\}^{\sigma}\left\{1+\left|\tilde{\lambda}_{j}\right|^{2}\right\}^{\sigma}} e^{\imath\left\langle x, \lambda_{j}-\tilde{\lambda}_{j}\right\rangle} \iint_{\left(\mathbb{R}^{2}\right)^{2}} d \xi_{j} d \tilde{\xi}_{j} \widehat{\rho}\left(\lambda_{j}-\xi_{j}\right) \widehat{\rho}\left(-\tilde{\lambda}_{j}+\tilde{\xi}_{j}\right) \mathcal{Q}\left(\xi_{j}, \tilde{\xi}_{j}\right) \\
& =\prod_{j=1}^{p-1} \iint_{\left(\mathbb{R}^{2}\right)^{2}} \frac{d \lambda_{j} d \tilde{\lambda}_{j}}{\left\{1+\left|\lambda_{j}\right|^{2}\right\}^{\sigma}\left\{1+\left|\tilde{\lambda}_{j}\right|^{2}\right\}^{\sigma}} \iint_{\left(\mathbb{R}^{2}\right)^{2}} d \xi_{j} d \tilde{\xi}_{j} \widehat{\rho}\left(\lambda_{j}-\xi_{j}\right) \widehat{\rho}\left(-\tilde{\lambda}_{j}+\tilde{\xi}_{j}\right) \mathcal{Q}\left(\xi_{j}, \tilde{\xi}_{j}\right) \\
& \int_{\mathbb{R}^{2}} \frac{d \lambda_{p}}{\left\{1+\left|\lambda_{p}\right|^{2}\right\}^{\sigma}\left\{1+\left|\lambda_{p}+\Sigma_{k=1}^{p-1}\left(\lambda_{k}-\tilde{\lambda}_{k}\right)\right|^{2}\right\}^{\sigma}} \\
& \iint_{\left(\mathbb{R}^{2}\right)^{2}} d \xi_{p} d \tilde{\xi}_{p} \widehat{\rho}\left(\lambda_{p}-\xi_{p}\right) \widehat{\rho}\left(-\lambda_{p}-\Sigma_{k=1}^{p-1}\left(\lambda_{k}-\tilde{\lambda}_{k}\right)+\tilde{\xi}_{p}\right) \mathcal{Q}\left(\xi_{p}, \tilde{\xi}_{p}\right),
\end{aligned}
$$


and so

$$
\begin{gathered}
\left|\int_{\mathbb{R}^{2}} d x \mathcal{R}(x)^{p}\right| \leq \prod_{j=1}^{p-1} \iint_{\left(\mathbb{R}^{2}\right)^{2}} d \xi_{j} d \tilde{\xi}_{j}\left|\mathcal{Q}\left(\xi_{j}, \tilde{\xi}_{j}\right)\right| \iint_{\left(\mathbb{R}^{2}\right)^{2}} \frac{d \lambda_{j} d \tilde{\lambda}_{j}}{\left\{1+\left|\xi_{j}+\lambda_{j}\right|^{2}\right\}^{\sigma}\left\{1+\left|\tilde{\xi}_{j}-\tilde{\lambda}_{j}\right|^{2}\right\}^{\sigma}}\left|\widehat{\rho}\left(\lambda_{j}\right)\right|\left|\widehat{\rho}\left(\tilde{\lambda}_{j}\right)\right| \\
\quad \iint_{\left(\mathbb{R}^{2}\right)^{2}} d \xi_{p} d \tilde{\xi}_{p}\left|\mathcal{Q}\left(\xi_{p}, \tilde{\xi}_{p}\right)\right| \\
\int_{\mathbb{R}^{2}} \frac{d \lambda_{p}}{\left\{1+\left|\xi_{p}+\lambda_{p}\right|^{2}\right\}^{\sigma}\left\{1+\left|\tilde{\xi}_{p}-\lambda_{p}-\Sigma_{k=1}^{p-1}\left(\lambda_{k}-\tilde{\lambda}_{k}\right)\right|^{2}\right\}^{\sigma}}\left|\widehat{\rho}\left(\lambda_{p}\right)\right|\left|\widehat{\rho}\left(\lambda_{p}+\Sigma_{k=1}^{p-1}\left(\lambda_{k}-\tilde{\lambda}_{k}\right)\right)\right| .
\end{gathered}
$$

The conclusion now comes from the fact that for all $\lambda, \xi \in \mathbb{R}^{2}$ and $\kappa>0$, one has

$$
\left|\hat{\rho}(\lambda)\left\{1+|\xi+\lambda|^{2}\right\}^{-\sigma}\right| \leq c_{\sigma, \rho, \kappa}\left\{1+|\lambda|^{2}\right\}^{-\kappa}\left\{1+|\xi|^{2}\right\}^{-\sigma} .
$$

Indeed, as $\rho$ is smooth and compactly-supported, one has, if $\sigma \geq 0$,

$$
\begin{aligned}
\mid \hat{\rho}(\lambda)\{1+\mid \xi+ & \left.\left.\lambda\right|^{2}\right\}^{-\sigma}|=| \hat{\rho}(\lambda)\left\{1+|\xi+\lambda|^{2}\right\}^{-\sigma}\left|\mathbf{1}_{\left\{|\lambda|>\frac{1}{2}|\xi|\right\}}+\right| \hat{\rho}(\lambda)\left\{1+|\xi+\lambda|^{2}\right\}^{-\sigma} \mid \mathbf{1}_{\left\{|\lambda|<\frac{1}{2}|\xi|\right\}} \\
& \leq c_{\sigma}\left[|\hat{\rho}(\lambda)| \mathbf{1}_{\left\{|\lambda|>\frac{1}{2}|\xi|\right\}}+|\hat{\rho}(\lambda)|\left\{1+|\xi|^{2}\right\}^{-\sigma} \mathbf{1}_{\left\{|\lambda|<\frac{1}{2}|\xi|\right\}}\right] \\
& \leq c_{\sigma, \rho, \kappa}\left[\left\{1+|\lambda|^{2}\right\}^{-\kappa}\left\{1+|\lambda|^{2}\right\}^{-\sigma} \mathbf{1}_{\left\{|\lambda|>\frac{1}{2}|\xi|\right\}}+\left\{1+|\lambda|^{2}\right\}^{-\kappa}\left\{1+|\xi|^{2}\right\}^{-\sigma} \mathbf{1}_{\left\{|\lambda|<\frac{1}{2}|\xi|\right\}}\right] \\
& \leq c_{\sigma, \rho, \kappa}\left\{1+|\lambda|^{2}\right\}^{-\kappa}\left\{1+|\xi|^{2}\right\}^{-\sigma},
\end{aligned}
$$

and we can exhibit similar bounds for $\sigma<0$.

\subsubsection{Convergence of the first component}

It actually corresponds to the result of [1, Proposition 1.2]. Let us only recall that the convergence is here a straightforward consequence of the elementary property

$$
\int_{\mathbb{R}^{2}} \frac{d \eta}{\left\{1+|\eta|^{2}\right\}^{\alpha}} K^{H}(\eta)<\infty
$$

valid for all $H=\left(H_{0}, H_{1}, H_{2}\right) \in(0,1)^{3}$ and $\alpha>\frac{3}{2}-\left(H_{0}+H_{1}+H_{2}\right)$.

\subsubsection{Convergence of the second component}

In this situation, let us first expand the expectation in the left-hand side of (39) (with $\tau=9$ ) as

$$
\begin{gathered}
\iint_{\left(\mathbb{R}^{2}\right)^{2}} \frac{d \lambda d \tilde{\lambda}}{\left\{1+|\lambda|^{2}\right\}^{\alpha}\left\{1+|\tilde{\lambda}|^{2}\right\}^{\alpha}} e^{\imath\langle x, \lambda-\tilde{\lambda}\rangle} \mathbb{E}\left[\left(\mathcal{F}_{x}(\rho) * \mathcal{F}_{x}\left(\mathrm{Q}_{s, t}^{n, m}\right)\right)(\lambda) \overline{\left(\mathcal{F}_{x}(\rho) * \mathcal{F}_{x}\left(\mathrm{Q}_{s, t}^{n, m}\right)\right)(\tilde{\lambda})}\right] \\
=\iint_{\left(\mathbb{R}^{2}\right)^{2}} \frac{d \lambda d \tilde{\lambda}}{\left\{1+|\lambda|^{2}\right\}^{\alpha}\left\{1+|\tilde{\lambda}|^{2}\right\}^{\alpha}} e^{\imath\langle x, \lambda-\tilde{\lambda}\rangle} \iint_{\left(\mathbb{R}^{2}\right)^{2}} d \xi d \tilde{\xi} \widehat{\rho}(\lambda-\xi) \widehat{\rho}(-\tilde{\lambda}+\tilde{\xi}) \\
\iint_{\left(\mathbb{R}^{2}\right)^{2}} d y d \tilde{y} e^{-\imath\langle\xi, y\rangle} e^{\imath\langle\tilde{\xi}, \tilde{y}\rangle} \mathbb{E}\left[Q \rho_{s, t}^{n, m}(y) \overline{Q_{s} \rho_{s, t}^{n, m}(\tilde{y})}\right] .
\end{gathered}
$$

Thanks to Lemma 3.2, we are thus left with the estimation of the quantity

$$
\mathcal{Q}_{Q} \odot \triangleq \iint_{\left(\mathbb{R}^{2}\right)^{2}} \frac{d \lambda d \tilde{\lambda}}{\left\{1+|\lambda|^{2}\right\}^{\alpha}\left\{1+|\tilde{\lambda}|^{2}\right\}^{\alpha}}\left|\iint_{\left(\mathbb{R}^{2}\right)^{2}} d y d \tilde{y} e^{-\imath\langle\lambda, y\rangle} e^{\imath\langle\tilde{\lambda}, \tilde{y}\rangle} \mathbb{E}\left[Q_{s, t}^{n, m}(y) \overline{Q_{s} \otimes_{s, t}^{n, m}(\tilde{y})}\right]\right| .
$$

Using Wick formula, it is easy to check that for every $j=1, \ldots, p$, the quantity

$$
\mathbb{E}\left[Q_{s, t}^{n, m}(y) \overline{\mathrm{Q}_{s, t}^{n, m}(\tilde{y})}\right]
$$


can be expanded as a sum of the form

$$
\sum_{(a, b) \in \mathcal{A}} c_{a, b} \mathbb{E}\left[?_{b_{1}}^{a_{1}}(y) \overline{?_{b_{2}}^{a_{2}}(\tilde{y})}\right] \mathbb{E}\left[?_{b_{3}}^{a_{3}}(y) \overline{?_{b_{4}}^{a_{4}}(\tilde{y})}\right]
$$

for some index set $\mathcal{A}$ such that $a_{i} \in\{n, m,\{n, m\}\}, b_{i} \in\{s, t,\{s, t\}\}$, and one has both $\left\{a_{1}, \ldots, a_{4}\right\} \cap$ $\{\{n, m\}\} \neq \emptyset$ and $\left\{b_{1}, \ldots, b_{4}\right\} \cap\{\{s, t\}\} \neq \emptyset$ (in other words, each of the summands contains at least one increment with respect to $(n, m)$ and one increment with respect to $(s, t))$.

By formula (34), each of the above summands can be written as

$$
\begin{aligned}
& \mathbb{E}\left[\varrho_{b_{1}}^{a_{1}}(y) \overline{q_{b_{2}}^{a_{2}}(\tilde{y})}\right] \mathbb{E}\left[\rho_{b_{3}}^{a_{3}}(y) \overline{?_{b_{4}}^{a_{4}}(\tilde{y})}\right] \\
& =\iint_{\left(\mathbb{R}^{2}\right)^{2}} d \eta d \tilde{\eta} e^{\imath\langle\eta, y\rangle} e^{-\imath\langle\eta, \tilde{y}\rangle} e^{\imath\langle\tilde{\eta}, y\rangle} e^{-\imath\langle\tilde{\eta}, \tilde{y}\rangle} L_{b_{1}, b_{2}}^{H,\left(a_{1}, a_{2}\right)}(\eta) L_{b_{3}, b_{4}}^{H,\left(a_{3}, a_{4}\right)}(\tilde{\eta}),
\end{aligned}
$$

and so

$$
\begin{aligned}
& \left|\iint_{\left(\mathbb{R}^{2}\right)^{2}} d y d \tilde{y} e^{-\imath\langle\lambda, y\rangle} e^{\imath\langle\tilde{\lambda}, \tilde{y}\rangle} \mathbb{E}\left[Q_{\diamond} \rho_{s, t}^{n, m}(y) \overline{Q_{\otimes} \rho_{s, t}^{n, m}(\tilde{y})}\right]\right| \\
& \leq \sum_{(a, b) \in \mathcal{A}} c_{a, b} \iint_{\left(\mathbb{R}^{2}\right)^{2}} d \eta d \tilde{\eta}\left|L_{b_{1}, b_{2}}^{H,\left(a_{1}, a_{2}\right)}(\eta)\right|\left|L_{b_{3}, b_{4}}^{H,\left(a_{3}, a_{4}\right)}(\tilde{\eta})\right| \delta_{\{\lambda=\eta+\tilde{\eta}\}} \delta_{\{\tilde{\lambda}=\eta+\tilde{\eta}\}} .
\end{aligned}
$$

Combining this bound with the bounds of Lemma 3.1, we can assert that for any $\varepsilon>0$ small enough,

$$
\begin{aligned}
& \mathcal{Q}_{Q_{0}} \lesssim 2^{-2 n \varepsilon}|t-s|^{2 \varepsilon} \\
& \qquad \iint_{\left(\mathbb{R}^{2}\right)^{2}} \frac{d \eta d \tilde{\eta}}{\left\{1+|\eta+\tilde{\eta}|^{2}\right\}^{2 \alpha}}\left\{K^{H_{\varepsilon, 0}}(\eta)+K^{H_{\varepsilon, 1}}(\eta)+K^{H_{\varepsilon, 2}}(\eta)\right\}\left\{K^{H_{\varepsilon, 0}}(\tilde{\eta})+K^{H_{\varepsilon, 1}}(\tilde{\eta})+K^{H_{\varepsilon, 2}}(\tilde{\eta})\right\} .
\end{aligned}
$$

The conclusion is then an immediate consequence of the following technical result:

Lemma 3.3. For all $H=\left(H_{0}, H_{1}, H_{2}\right), \tilde{H}=\left(\tilde{H}_{0}, \tilde{H}_{1}, \tilde{H}_{2}\right) \in(0,1)^{3}$ satisfying

$$
0<H_{1}, \tilde{H}_{1}<\frac{3}{4} \quad, \quad 0<H_{2}, \tilde{H}_{2}<\frac{3}{4} \quad, \quad 1<H_{0}+H_{1}+H_{2} \leq \frac{5}{4} \quad, \quad 1<\tilde{H}_{0}+\tilde{H}_{1}+\tilde{H}_{2} \leq \frac{5}{4},
$$

and any

$$
\alpha \in\left(\max \left(\frac{3}{2}-\left(H_{0}+H_{1}+H_{2}\right), \frac{3}{2}-\left(\tilde{H}_{0}+\tilde{H}_{1}+\tilde{H}_{2}\right)\right), \frac{1}{2}\right),
$$

it holds that

$$
\iint_{\left(\mathbb{R}^{2}\right)^{2}} \frac{d \eta d \tilde{\eta}}{\left\{1+|\eta-\tilde{\eta}|^{2}\right\}^{2 \alpha}} K^{H}(\eta) K^{\tilde{H}}(\tilde{\eta})<\infty
$$

\section{Proof.}

Step 0: Simplification of the problem. Let us show that the problem actually reduces to the consideration of the four following integrals:

$$
\begin{gathered}
\mathcal{J}_{1} \triangleq \int_{\mathbb{R}^{2}} d \eta \int_{\mathbb{R}^{2}} d \tilde{\eta} \frac{1}{\left\{1+|\eta|^{2}\right\}^{\alpha}} \frac{1}{\left\{1+|\tilde{\eta}|^{2}\right\}^{\alpha}} K^{H}(\eta) K^{\tilde{H}}(\tilde{\eta}), \\
\mathcal{J}_{2} \triangleq \int_{0}^{\infty} d \eta_{1} \int_{0}^{\infty} d \tilde{\eta}_{1} \int_{0}^{\infty} d \eta_{2} \int_{\eta_{2}}^{2 \eta_{2}} d \tilde{\eta}_{2} \frac{1}{\left\{1+\eta_{1}^{2}+\left(\eta_{2}-\tilde{\eta}_{2}\right)^{2}\right\}^{\alpha}} \frac{1}{\left\{1+\tilde{\eta}_{1}^{2}+\left(\eta_{2}-\tilde{\eta}_{2}\right)^{2}\right\}^{\alpha}} K^{H}(\eta) K^{\tilde{H}}(\tilde{\eta}), \\
\text { imsart-aihp ver. 2014/10/16 file: quadratic-wave-rougher-accepted-version.tex date: February 14, } 2019
\end{gathered}
$$




$$
\mathcal{J}_{3} \triangleq \int_{0}^{\infty} d \eta_{1} \int_{\eta_{1}}^{2 \eta_{1}} d \tilde{\eta}_{1} \int_{0}^{\infty} d \eta_{2} \int_{\eta_{2}}^{2 \eta_{2}} d \tilde{\eta}_{2} \frac{1}{\left\{1+|\eta-\tilde{\eta}|^{2}\right\}^{2 \alpha}} K^{H}(\eta) K^{\tilde{H}}(\tilde{\eta})
$$

and

$$
\mathcal{J}_{4} \triangleq \int_{0}^{\infty} d \eta_{1} \int_{\eta_{1}}^{2 \eta_{1}} d \tilde{\eta}_{1} \int_{0}^{\infty} d \tilde{\eta}_{2} \int_{\tilde{\eta}_{2}}^{2 \tilde{\eta}_{2}} d \eta_{2} \frac{1}{\left\{1+|\eta-\tilde{\eta}|^{2}\right\}^{2 \alpha}} K^{H}(\eta) K^{\tilde{H}}(\tilde{\eta})
$$

First, observe that for obvious symmetric and sign reasons, we can focus on the integration over the two domains $\mathcal{D}_{1} \triangleq\left\{\eta_{1}<0<\tilde{\eta}_{1},\left(\eta_{2}, \tilde{\eta}_{2}\right) \in \mathbb{R}^{2}\right\}$ and $\mathcal{D}_{2} \triangleq\left\{0<\eta_{1}<\tilde{\eta}_{1},\left(\eta_{2}, \tilde{\eta}_{2}\right) \in \mathbb{R}^{2}\right\}$.

As far as $\mathcal{D}_{1}$ is concerned, let us decompose the domain as $\mathcal{D}_{1}=\mathcal{D}_{1}^{1} \cup \mathcal{D}_{1}^{2}$, with $\mathcal{D}_{1}^{1} \triangleq\left\{\eta_{1}<0<\tilde{\eta}_{1}, \eta_{2} \tilde{\eta}_{2}<\right.$ $0\}$ and $\mathcal{D}_{1}^{2} \triangleq\left\{\eta_{1}<0<\tilde{\eta}_{1}, \eta_{2} \tilde{\eta}_{2} \geq 0\right\}$. For $(\eta, \tilde{\eta}) \in \mathcal{D}_{1}^{1}$, one has $\left|\eta_{i}-\tilde{\eta}_{i}\right|^{2} \geq \max \left(\left|\eta_{i}\right|^{2},\left|\tilde{\eta}_{i}\right|^{2}\right)$ for $i \in\{1,2\}$, and so the integral over $\mathcal{D}_{1}^{1}$ is bounded by $\mathcal{J}_{1}$. For $(\eta, \tilde{\eta}) \in \mathcal{D}_{1}^{2}$, one has again $\left|\eta_{1}-\tilde{\eta}_{1}\right|^{2} \geq \max \left(\left|\eta_{1}\right|^{2},\left|\tilde{\eta}_{1}\right|^{2}\right)$, as well as one of the following four situations: $\left|\eta_{2}\right| \geq 2\left|\tilde{\eta}_{2}\right|,\left|\tilde{\eta}_{2}\right| \geq 2\left|\eta_{2}\right|,\left|\eta_{2}\right| \leq\left|\tilde{\eta}_{2}\right| \leq 2\left|\eta_{2}\right|$ or $\left|\tilde{\eta}_{2}\right| \leq\left|\eta_{2}\right| \leq 2\left|\tilde{\eta}_{2}\right|$. In the first two cases, one has $\left|\eta_{2}-\tilde{\eta}_{2}\right|^{2} \geq \frac{1}{4} \max \left(\left|\eta_{2}\right|^{2},\left|\tilde{\eta}_{2}\right|^{2}\right)$ and so we can again go back to the integral $\mathcal{J}_{1}$, while the integration in the third and fourth cases clearly reduces to the consideration of $\mathcal{J}_{2}$.

Along the same ideas, decompose $\mathcal{D}_{2}$ into $\mathcal{D}_{2}=\mathcal{D}_{2}^{1} \cup \mathcal{D}_{2}^{2}$, with $\mathcal{D}_{2}^{1} \triangleq\left\{0<\eta_{1}<\tilde{\eta}_{1}<2 \eta_{1},\left(\eta_{2}, \tilde{\eta}_{2}\right) \in \mathbb{R}^{2}\right\}$ and $\mathcal{D}_{2}^{2} \triangleq\left\{0<2 \eta_{1}<\tilde{\eta}_{1},\left(\eta_{2}, \tilde{\eta}_{2}\right) \in \mathbb{R}^{2}\right\}$. For $(\eta, \tilde{\eta}) \in \mathcal{D}_{2}^{1}$, one has either: $(i) \eta_{2} \tilde{\eta}_{2}>0$ and $0<\left|\eta_{2}\right|<$ $\left|\tilde{\eta}_{2}\right|<2\left|\eta_{2}\right|$, which leads us to the consideration of $\mathcal{J}_{3}$; (ii) $\eta_{2} \tilde{\eta}_{2}>0$ and $0<\left|\tilde{\eta}_{2}\right|<\left|\eta_{2}\right|<2\left|\tilde{\eta}_{2}\right|$, which leads us to the consideration of $\mathcal{J}_{4}$; (iii) $\eta_{2} \tilde{\eta}_{2}>0$ and $\left(0<2\left|\tilde{\eta}_{2}\right|<\left|\eta_{2}\right|\right.$ or $\left.0<2\left|\eta_{2}\right|<\left|\tilde{\eta}_{2}\right|\right)$, so that $\left|\eta_{2}-\tilde{\eta}_{2}\right|^{2} \geq \frac{1}{4} \max \left(\left|\eta_{2}\right|^{2},\left|\tilde{\eta}_{2}\right|^{2}\right)$, which, by inverting the roles of $\left(\eta_{1}, \tilde{\eta}_{1}\right)$ and $\left(\eta_{2}, \tilde{\eta}_{2}\right)$, brings us back to $\mathcal{J}_{2}$; (iv) $\eta_{2} \tilde{\eta}_{2}<0$, so that $\left|\eta_{2}-\tilde{\eta}_{2}\right|^{2} \geq \max \left(\left|\eta_{2}\right|^{2},\left|\tilde{\eta}_{2}\right|^{2}\right)$, and we are again brought back to $\mathcal{J}_{2}$. Finally, if $(\eta, \tilde{\eta}) \in \mathcal{D}_{2}^{2}$, then $\left|\eta_{1}-\tilde{\eta}_{1}\right|^{2} \geq \frac{1}{4} \max \left(\left|\eta_{1}\right|^{2},\left|\tilde{\eta}_{1}\right|^{2}\right)$ and we can use the same splitting as above for $\left(\eta_{2}, \tilde{\eta}_{2}\right)$ in order to reduce the problem to the consideration of either $\mathcal{J}_{1}$ or $\mathcal{J}_{2}$.

Step 1: Estimation of $\mathcal{J}_{1}$. The quantity under consideration here can of course be written as

$$
\mathcal{J}_{1}=\left(\int_{\mathbb{R}^{2}} \frac{d \eta}{\left\{1+|\eta|^{2}\right\}^{\alpha}} K^{H}(\eta)\right)\left(\int_{\mathbb{R}^{2}} \frac{d \tilde{\eta}}{\left\{1+|\tilde{\eta}|^{2}\right\}^{\alpha}} K^{\tilde{H}}(\tilde{\eta})\right)
$$

and we can thus conclude with the first-order statement (40). 
Step 2: Estimation of $\mathcal{J}_{2}$. One has here

$$
\begin{aligned}
& \mathcal{J}_{2}=\int_{0}^{\infty} d \eta_{1} \int_{0}^{\infty} d \tilde{\eta}_{1} \int_{0}^{\infty} d \eta_{2} \eta_{2} \int_{0}^{1} d r \frac{1}{\left\{1+\eta_{1}^{2}+\eta_{2}^{2} r^{2}\right\}^{\alpha}} \frac{1}{\left\{1+\tilde{\eta}_{1}^{2}+\eta_{2}^{2} r^{2}\right\}^{\alpha}} \\
& \frac{\left|\eta_{1}\right|^{1-2 H_{1}}\left|\tilde{\eta}_{1}\right|^{1-2 \tilde{H}_{1}}}{\left|\eta_{2}\right|^{2\left(H_{2}+\tilde{H}_{2}\right)-2}(1+r)^{2 \tilde{H}_{2}-1}} \frac{1}{\left\{1+|\eta|^{1+2 H_{0}}\right\}} \frac{1}{\left\{1+\left(\tilde{\eta}_{1}^{2}+\eta_{2}^{2}(1+r)^{2}\right)^{\tilde{H}_{0}+\frac{1}{2}}\right\}} \\
& \lesssim \int_{0}^{\infty} d \eta_{1} \int_{0}^{\infty} d \tilde{\eta}_{1} \int_{0}^{\infty} d \eta_{2} \frac{\left|\eta_{1}\right|^{1-2 H_{1}}\left|\tilde{\eta}_{1}\right|^{1-2 \tilde{H}_{1}}}{\left|\eta_{2}\right|^{2\left(H_{2}+\tilde{H}_{2}\right)-3}} \frac{1}{\left\{1+|\eta|^{1+2 H_{0}}\right\}\left\{1+\left(\tilde{\eta}_{1}^{2}+\eta_{2}^{2}\right)^{\tilde{H}_{0}+\frac{1}{2}}\right\}} \\
& \int_{0}^{1} d r \frac{1}{\left\{1+\eta_{1}^{2}+\eta_{2}^{2} r^{2}\right\}^{\alpha}} \frac{1}{\left\{1+\tilde{\eta}_{1}^{2}+\eta_{2}^{2} r^{2}\right\}^{\alpha}} \\
& \lesssim \int_{0}^{\infty} \frac{d \eta_{2}}{\left|\eta_{2}\right|^{2\left(H_{2}+\tilde{H}_{2}\right)-3}}\left[\int_{0}^{\infty} \frac{d \eta_{1}}{\left|\eta_{1}\right|^{2 H_{1}-1}\left\{1+\left(\eta_{1}^{2}+\eta_{2}^{2}\right)^{H_{0}+\frac{1}{2}}\right\}}\left(\int_{0}^{1} \frac{d r}{\left\{1+\eta_{1}^{2}+\eta_{2}^{2} r^{2}\right\}^{2 \alpha}}\right)^{\frac{1}{2}}\right] \\
& {\left[\int_{0}^{\infty} \frac{d \tilde{\eta}_{1}}{\left|\tilde{\eta}_{1}\right|^{2 \tilde{H}_{1}-1}\left\{1+\left(\tilde{\eta}_{1}^{2}+\eta_{2}^{2}\right)^{\tilde{H}_{0}+\frac{1}{2}}\right\}}\left(\int_{0}^{1} \frac{d r}{\left\{1+\tilde{\eta}_{1}^{2}+\eta_{2}^{2} r^{2}\right\}^{2 \alpha}}\right)^{\frac{1}{2}}\right]} \\
& \lesssim \int_{0}^{\infty} \frac{d \eta_{2}}{\left|\eta_{2}\right|^{2\left(H_{2}+\tilde{H}_{2}\right)-3}}\left(\int_{0}^{\infty} \frac{d \eta_{1}}{\left|\eta_{1}\right|^{4 H_{1}-2}\left\{1+\left(\eta_{1}^{2}+\eta_{2}^{2}\right)^{2 H_{0}+1}\right\}}\right)^{\frac{1}{2}} \\
& \left(\int_{0}^{\infty} \frac{d \tilde{\eta}_{1}}{\left|\tilde{\eta}_{1}\right|^{4 \tilde{H}_{1}-2}\left\{1+\left(\tilde{\eta}_{1}^{2}+\eta_{2}^{2}\right)^{2 \tilde{H}_{0}+1}\right\}}\right)^{\frac{1}{2}}\left(\int_{0}^{\infty} d \lambda \int_{0}^{1} \frac{d r}{\left\{1+\lambda^{2}+\eta_{2}^{2} r^{2}\right\}^{2 \alpha}}\right) \\
& \lesssim \int_{0}^{\infty} \frac{d \eta_{2}}{\left|\eta_{2}\right|^{2\left(H_{2}+\tilde{H}_{2}\right)-2}}\left(\int_{0}^{\infty} \frac{d \eta_{1}}{\left|\eta_{1}\right|^{4 H_{1}-2}\left\{1+\left(\eta_{1}^{2}+\eta_{2}^{2}\right)^{2 H_{0}+1}\right\}}\right)^{\frac{1}{2}} \\
& \left(\int_{0}^{\infty} \frac{d \tilde{\eta}_{1}}{\left|\tilde{\eta}_{1}\right|^{4 \tilde{H}_{1}-2}\left\{1+\left(\tilde{\eta}_{1}^{2}+\eta_{2}^{2}\right)^{2 \tilde{H}_{0}+1}\right\}}\right)^{\frac{1}{2}}\left(\int_{0}^{\infty} d \lambda \int_{0}^{\eta_{2}} \frac{d r}{\left\{1+\lambda^{2}+r^{2}\right\}^{2 \alpha}}\right) \\
& \lesssim\left(\int_{0}^{\infty} \frac{d \eta_{2}}{\left|\eta_{2}\right|^{4 H_{2}-2}} \int_{0}^{\infty} \frac{d \eta_{1}}{\left|\eta_{1}\right|^{4 H_{1}-2}\left\{1+|\eta|^{4 H_{0}+2}\right\}}\left(\int_{0}^{\infty} d \lambda \int_{0}^{\eta_{2}} \frac{d r}{\left\{1+\lambda^{2}+r^{2}\right\}^{2 \alpha}}\right)\right)^{\frac{1}{2}} \\
& \left(\int_{0}^{\infty} \frac{d \tilde{\eta}_{2}}{\left|\tilde{\eta}_{2}\right|^{\mid \tilde{H}_{2}-2}} \int_{0}^{\infty} \frac{d \tilde{\eta}_{1}}{\left|\tilde{\eta}_{1}\right|^{4 \tilde{H}_{1}-2}\left\{1+|\tilde{\eta}|^{4 \tilde{H}_{0}+2}\right\}}\left(\int_{0}^{\infty} d \lambda \int_{0}^{\tilde{\eta}_{2}} \frac{d r}{\left\{1+\lambda^{2}+r^{2}\right\}^{2 \alpha}}\right)\right)^{\frac{1}{2}} .
\end{aligned}
$$

At this point, let us pick $\varepsilon>0$ such that $2 \alpha-\frac{1}{2}-\varepsilon>0$ (noting that $\alpha>\frac{1}{4}$ by (45)) and write

$$
\int_{0}^{\infty} d \lambda \int_{0}^{\eta_{2}} \frac{d r}{\left\{1+\lambda^{2}+r^{2}\right\}^{2 \alpha}} \leq \int_{0}^{\infty} \frac{d \lambda}{\left\{1+\lambda^{2}\right\}^{\frac{1}{2}+\varepsilon}} \int_{0}^{\eta_{2}} \frac{d r}{\left\{1+r^{2}\right\}^{2 \alpha-\frac{1}{2}-\varepsilon}} \lesssim 1+\left|\eta_{2}\right|^{2-4 \alpha+2 \varepsilon},
$$

so that

$$
\begin{aligned}
& \int_{0}^{\infty} \frac{d \eta_{2}}{\left|\eta_{2}\right|^{4 H_{2}-2}} \int_{0}^{\infty} \frac{d \eta_{1}}{\left|\eta_{1}\right|^{4 H_{1}-2}\left\{1+|\eta|^{4 H_{0}+2}\right\}}\left(\int_{0}^{\infty} d \lambda \int_{0}^{\eta_{2}} \frac{d r}{\left\{1+\lambda^{2}+r^{2}\right\}^{2 \alpha}}\right) \\
& \lesssim \int_{0}^{\infty} \frac{d \eta_{1}}{\left|\eta_{1}\right|^{4 H_{1}-2}\left\{1+\left|\eta_{1}\right|^{4 H_{0}+2}\right\}} \int_{0}^{1} \frac{d \eta_{2}}{\left|\eta_{2}\right|^{4 H_{2}-2}}+\int_{0}^{\infty} d \eta_{1} \int_{1}^{\infty} d \eta_{2} \frac{1}{\left|\eta_{1}\right|^{4 H_{1}-2}\left|\eta_{2}\right|^{4 H_{2}+4 \alpha-4-2 \varepsilon}|\eta|^{4 H_{0}+2}}
\end{aligned}
$$

Using conditions (45)-(46), it is easy to check that the latter integrals are finite for any $\varepsilon>0$ small enough. Note in particular that, since $H_{0}+H_{1}+H_{2}>1$ and $0<H_{2}<\frac{3}{4}$, one has $H_{0}+H_{1}>\frac{1}{4}$, which guarantees the convergence of the first integral.

It is then clear that these arguments also apply to the second term in (48), which achieves to prove that $\mathcal{J}_{2}$ is finite. 
Step 3: Estimation of $\mathcal{J}_{3}$. Let us write

$$
\begin{aligned}
\mathcal{J}_{3}= & \int_{0}^{\infty} d \eta_{1} \int_{0}^{\infty} d \eta_{2} \eta_{1} \eta_{2} \int_{0}^{1} d r_{1} \int_{0}^{1} d r_{2} \frac{1}{\left\{1+\eta_{1}^{2} r_{1}^{2}+\eta_{2}^{2} r_{2}^{2}\right\}^{2 \alpha}} \\
& \frac{\left(1+r_{1}\right)^{1-2 \tilde{H}_{1}}\left(1+r_{2}\right)^{1-2 \tilde{H}_{2}}}{\left|\eta_{1}\right|^{2\left(H_{1}+\tilde{H}_{1}\right)-2}\left|\eta_{2}\right|^{2\left(H_{2}+\tilde{H}_{2}\right)-2}} \frac{1}{1+|\eta|^{2 H_{0}+1}} \frac{1}{1+\left(\eta_{1}^{2}\left(1+r_{1}\right)^{2}+\eta_{2}^{2}\left(1+r_{2}\right)^{2}\right)^{\tilde{H}_{0}+\frac{1}{2}}} \\
\lesssim & \int_{0}^{\infty} \int_{0}^{\infty} \frac{d \eta_{1} d \eta_{2}}{\left|\eta_{1}\right|^{2\left(H_{1}+\tilde{H}_{1}\right)-2}\left|\eta_{2}\right|^{2\left(H_{2}+\tilde{H}_{2}\right)-2}\left\{1+|\eta|^{2\left(H_{0}+\tilde{H}_{0}\right)+2}\right\}} \int_{0}^{\eta_{1}} d r_{1} \int_{0}^{\eta_{2}} d r_{2} \frac{1}{\left\{1+r_{1}^{2}+r_{2}^{2}\right\}^{2 \alpha}} \\
\lesssim & \int_{0<\eta_{1}, \eta_{2}<1} \frac{d \eta_{1} d \eta_{2}}{\left|\eta_{1}\right|^{2\left(H_{1}+\tilde{H}_{1}\right)-3}\left|\eta_{2}\right|^{2\left(H_{2}+\tilde{H}_{2}\right)-3}} \\
& +\int_{\substack{0<\eta_{1}, \eta_{2}<\infty \\
\eta_{1} \vee \eta_{2}>1}} \frac{d \eta_{1} d \eta_{2}}{\left|\eta_{1}\right|^{2\left(H_{1}+\tilde{H}_{1}\right)-2}\left|\eta_{2}\right|^{2\left(H_{2}+\tilde{H}_{2}\right)-2}\left\{1+|\eta|^{2\left(H_{0}+\tilde{H}_{0}\right)+2}\right\}} \iint_{\left[0, \eta_{1} \vee \eta_{2}\right]^{2}} \frac{d r_{1} d r_{2}}{\left\{1+r_{1}^{2}+r_{2}^{2}\right\}^{2 \alpha}}
\end{aligned}
$$

The first integral is clearly finite. Then, since $\alpha \in\left(\frac{1}{4}, \frac{1}{2}\right)$,

$$
\iint_{\left[0, \eta_{1} \vee \eta_{2}\right]^{2}} \frac{d r_{1} d r_{2}}{\left\{1+r_{1}^{2}+r_{2}^{2}\right\}^{2 \alpha}} \lesssim \int_{0}^{2\left(\eta_{1} \vee \eta_{2}\right)} d \rho \frac{\rho}{\left\{1+\rho^{2}\right\}^{2 \alpha}} \lesssim 1+\left(\eta_{1} \vee \eta_{2}\right)^{2-4 \alpha},
$$

and so, using the fact that $H_{i}, \tilde{H}_{i} \in\left(0, \frac{3}{4}\right)$ for $i \in\{1,2\}$, we get

$$
\begin{gathered}
\int_{\substack{0<\eta_{1}, \eta_{2}<\infty \\
\eta_{1} \vee \eta_{2}>1}} \frac{d \eta_{1} d \eta_{2}}{\left|\eta_{1}\right|^{2\left(H_{1}+\tilde{H}_{1}\right)-2}\left|\eta_{2}\right|^{2\left(H_{2}+\tilde{H}_{2}\right)-2}\left\{1+|\eta|^{2\left(H_{0}+\tilde{H}_{0}\right)+2}\right\}} \iint_{\left[0, \eta_{1} \vee \eta_{2}\right]^{2}} \frac{d r_{1} d r_{2}}{\left\{1+r_{1}^{2}+r_{2}^{2}\right\}^{2 \alpha}} \\
\lesssim \int_{1}^{\infty} \frac{d r}{r^{2\left(H_{0}+H_{1}+H_{2}\right)+2\left(\tilde{H}_{0}+\tilde{H}_{1}+\tilde{H}_{2}\right)+4 \alpha-5}} .
\end{gathered}
$$

Thanks to (46), we can assert that the latter integral is finite, and accordingly $\mathcal{J}_{3}$ is finite too.

Step 4: Estimation of $\mathcal{J}_{4}$. We have of course

$$
\mathcal{J}_{4}=\int_{0}^{\infty} d \eta_{1} \int_{\eta_{1}}^{2 \eta_{1}} d \tilde{\eta}_{1} \int_{0}^{\infty} d \eta_{2} \int_{\frac{\eta_{2}}{2}}^{\eta_{2}} d \tilde{\eta}_{2} \frac{1}{\left\{1+|\eta-\tilde{\eta}|^{2}\right\}^{2 \alpha}} K^{H}(\eta) K^{H}(\tilde{\eta}),
$$

and from there it is easy to mimic the arguments that we have used for $\mathcal{J}_{3}$.

\subsubsection{Convergence of the third component}

Noting that

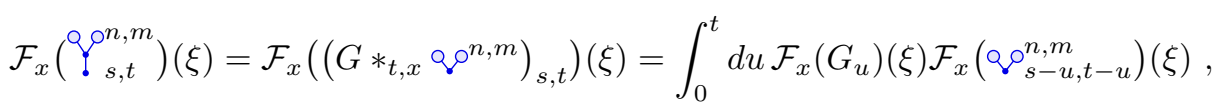

we get

$$
\begin{aligned}
& \mathbb{E}\left[\left|\mathcal{F}_{x}^{-1}\left(\left\{1+|\cdot|^{2}\right\}^{\frac{1}{2}(1-2 \alpha)} \mathcal{F}_{x}\left(\rho \cdot \mathbf{Q}_{s, t}^{n, m}\right)\right)(x)\right|^{2}\right] \\
& =\iint_{\left(\mathbb{R}^{2}\right)^{2}} \frac{d \lambda d \tilde{\lambda}}{\left\{1+|\lambda|^{2}\right\}^{\frac{1}{2}(2 \alpha-1)}\left\{1+|\tilde{\lambda}|^{2}\right\}^{\frac{1}{2}(2 \alpha-1)}} e^{\imath\langle x, \lambda-\tilde{\lambda}\rangle} \iint_{\left(\mathbb{R}^{2}\right)^{2}} d \xi d \tilde{\xi} \widehat{\rho}(\lambda-\xi) \widehat{\rho}(-\tilde{\lambda}+\tilde{\xi}) \\
& \quad \iint_{[0, t]^{2}} d u d \tilde{u} \mathcal{F}_{x}\left(G_{u}\right)(\xi) \overline{\mathcal{F}_{x}\left(G_{\tilde{u}}\right)(\tilde{\xi})} \iint_{\left(\mathbb{R}^{2}\right)^{2}} d y d \tilde{y} e^{-\imath\langle\xi, y\rangle} e^{\imath\langle\tilde{\xi}, \tilde{y}\rangle} \mathbb{E}\left[\otimes_{s-u, t-u}^{n, m}(y) \overline{Q_{s} \rho_{s-\tilde{u}, t-\tilde{u}}^{n, m}(\tilde{y})}\right],
\end{aligned}
$$


and so, thanks to Lemma 3.2 , we only need to estimate the quantity

$$
\begin{aligned}
& \mathcal{Q}_{Q} \triangleq \| \iint_{\left(\mathbb{R}^{2}\right)^{2}} \frac{d \lambda d \tilde{\lambda}}{\left\{1+|\lambda|^{2}\right\}^{\frac{1}{2}(2 \alpha-1)}\left\{1+|\tilde{\lambda}|^{2}\right\}^{\frac{1}{2}(2 \alpha-1)}} \\
& \iint_{[0, t]^{2}} d u d \tilde{u}\left|\mathcal{F}_{x}\left(G_{u}\right)(\lambda)\right|\left|\mathcal{F}_{x}\left(G_{\tilde{u}}\right)(\tilde{\lambda})\right|\left|\iint_{\left(\mathbb{R}^{2}\right)^{2}} d y d \tilde{y} e^{-\imath\langle\lambda, y\rangle} e^{\imath\langle\tilde{\lambda}, \tilde{y}\rangle} \mathbb{E}\left[Q \rho_{s-u, t-u}^{n, m}(y) \overline{Q_{\odot} \rho_{s-\tilde{u}, t-\tilde{u}}^{n, m}(\tilde{y})}\right]\right| .
\end{aligned}
$$

To this end, we can first use the bound (43), together with the estimates of Lemma 3.1, to deduce that, for any $\varepsilon>0$ small enough,

$$
\begin{array}{r}
\mathcal{Q}_{\underline{Q} \rho} \lesssim 2^{-2 n \varepsilon}|t-s|^{2 \varepsilon} \iint_{\left(\mathbb{R}^{2}\right)^{2}} \frac{d \eta d \tilde{\eta}}{\left\{1+|\eta+\tilde{\eta}|^{2}\right\}^{2 \alpha-1}} \iint_{[0, t]^{2}} d u d \tilde{u}\left|\mathcal{F}_{x}\left(G_{u}\right)(\eta+\tilde{\eta})\right|\left|\mathcal{F}_{x}\left(G_{\tilde{u}}\right)(\eta+\tilde{\eta})\right| \\
\left\{K^{H_{\varepsilon, 0}}(\eta)+K^{H_{\varepsilon, 1}}(\eta)+K^{H_{\varepsilon, 2}}(\eta)\right\}\left\{K^{H_{\varepsilon, 0}}(\tilde{\eta})+K^{H_{\varepsilon, 1}}(\tilde{\eta})+K^{H_{\varepsilon, 2}}(\tilde{\eta})\right\}
\end{array}
$$

Using the elementary estimate

$$
\sup _{u \in[0,1]}\left|\mathcal{F}_{x}\left(G_{u}\right)(\eta+\tilde{\eta})\right| \lesssim\left\{1+|\eta+\tilde{\eta}|^{2}\right\}^{-\frac{1}{2}},
$$

we have thus, for any $\varepsilon>0$ small enough,

$$
\begin{aligned}
\mathcal{Q}_{Q} \rho & 2^{-2 n \varepsilon}|t-s|^{2 \varepsilon} \\
& \iint_{\left(\mathbb{R}^{2}\right)^{2}} \frac{d \eta d \tilde{\eta}}{\left\{1+|\eta+\tilde{\eta}|^{2}\right\}^{2 \alpha}}\left\{K^{H_{\varepsilon, 0}}(\eta)+K^{H_{\varepsilon, 1}}(\eta)+K^{H_{\varepsilon, 2}}(\eta)\right\}\left\{K^{H_{\varepsilon, 0}}(\tilde{\eta})+K^{H_{\varepsilon, 1}}(\tilde{\eta})+K^{H_{\varepsilon, 2}}(\tilde{\eta})\right\} .
\end{aligned}
$$

Observe that we are here in the very same position as in (44), and so, using the same technical Lemma 3.3, we get the desired estimate (39) for $\tau=$ ?

\subsubsection{Convergence of the fourth component}

First, observe that $\mathcal{F}_{x}\left(\wp_{s, t}^{n, m}\right)$ can be readily expanded as

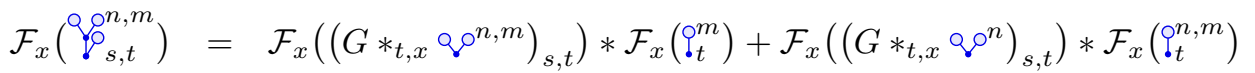

$$
\begin{aligned}
& +\mathcal{F}_{x}\left(\left(G *_{t, x} \mathrm{Q} \rho^{n, m}\right)_{s}\right) * \mathcal{F}_{x}\left(?_{s, t}^{m}\right)+\mathcal{F}_{x}\left(\left(G *_{t, x} \mathrm{Q}^{n}\right)_{s}\right) * \mathcal{F}_{x}\left(\varrho_{s, t}^{n, m}\right) \text {. }
\end{aligned}
$$

As it should be clear to the reader, the subsequent arguments could be applied to any of these four terms, and thus we will only focus on the estimate related to

$$
\begin{aligned}
& \mathbb{E}\left[\left|\mathcal{F}_{x}^{-1}\left(\left\{1+|.|^{2}\right\}^{-\frac{\alpha}{2}}\left(\mathcal{F}_{x}(\rho) *\left(\mathcal{F}_{x}\left(\left(G * Q \rho^{n, m}\right)_{s, t}\right) * \mathcal{F}_{x}\left(\varrho_{\bullet}^{m}\right)\right)\right)\right)(x)\right|^{2}\right] \\
& =\iint_{\left(\mathbb{R}^{2}\right)^{2}} \frac{d \lambda d \tilde{\lambda}}{\left\{1+|\lambda|^{2}\right\}^{\frac{\alpha}{2}}\left\{1+|\tilde{\lambda}|^{2}\right\}^{\frac{\alpha}{2}}} e^{\imath\langle x, \lambda-\tilde{\lambda}\rangle} \iint_{\left(\mathbb{R}^{2}\right)^{2}} d \xi d \tilde{\xi} \widehat{\rho}(\lambda-\xi) \widehat{\rho}(-\tilde{\lambda}+\tilde{\xi}) \\
& \iint_{\left(\mathbb{R}^{2}\right)^{2}} d \beta d \tilde{\beta} \iint_{[0, t]^{2}} d u d \tilde{u} \mathcal{F}_{x}\left(G_{u}\right)(\beta) \overline{\mathcal{F}_{x}\left(G_{\tilde{u}}\right)(\tilde{\beta})}
\end{aligned}
$$

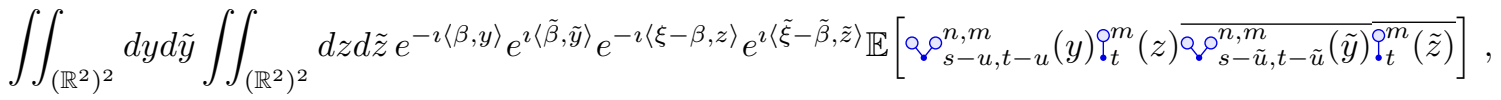


which, by Lemma 3.2, reduces to bounding the quantity

$$
\begin{aligned}
& \mathcal{Q}_{\mathfrak{\wp}} \triangleq \iint_{\left(\mathbb{R}^{2}\right)^{2}} \frac{d \lambda d \tilde{\lambda}}{\left\{1+|\lambda|^{2}\right\}^{\frac{\alpha}{2}}\left\{1+|\tilde{\lambda}|^{2}\right\}^{\frac{\alpha}{2}}} \\
& \iint_{\left(\mathbb{R}^{2}\right)^{2}} d \beta d \tilde{\beta} \iint_{[0, t]^{2}} d u d \tilde{u} \mathcal{F}_{x}\left(G_{u}\right)(\beta) \overline{\mathcal{F}_{x}\left(G_{\tilde{u}}\right)(\tilde{\beta})}
\end{aligned}
$$

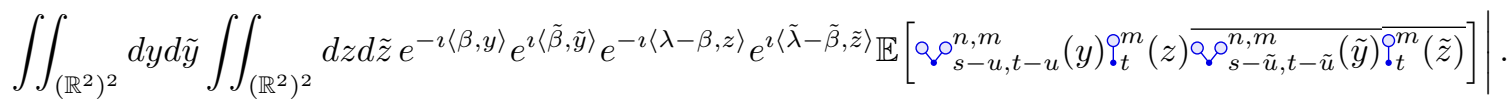

Using Wick formula, we can expand (along the same idea as in Section 3.1.2) the expectation

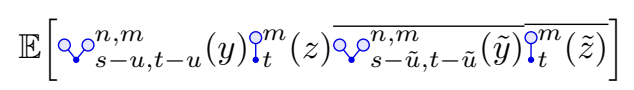

as a sum of terms of the form

$$
\begin{aligned}
& c_{a ; b}^{1} \mathbb{E}\left[\varphi_{b_{1}}^{a_{1}}(y) \overline{\varrho_{b_{2}}^{a_{2}}(\tilde{y})}\right] \mathbb{E}\left[\varphi_{b_{3}}^{a_{3}}(y) \overline{?_{b_{4}}^{a_{4}}(\tilde{y})}\right] \mathbb{E}\left[\varrho_{t}^{m}(z) \overline{\varrho_{t}^{m}(\tilde{z})}\right],
\end{aligned}
$$

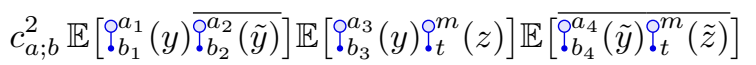

or

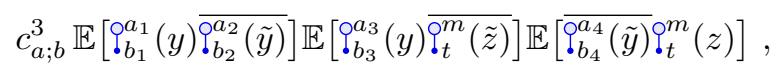

where $a_{i} \in\{n, m,\{n, m\}\}, b_{1}, b_{3} \in\{s-u, t-u,\{s-u, t-u\}\}, b_{2}, b_{4} \in\{s-\tilde{u}, t-\tilde{u},\{s-\tilde{u}, t-\tilde{u}\}\}$, and one has both $\left\{a_{1}, \ldots, a_{4}\right\} \cap\{\{n, m\}\} \neq \emptyset$ and $\left\{b_{1}, \ldots, b_{4}\right\} \cap(\{\{s-u, t-u\},\{s-\tilde{u}, t-\tilde{u}\}\}) \neq \emptyset$. An example of a pair $(a ; b)$ satisfying these conditions is given by

$$
(a ; b)=((\{n, m\}, m, m, m) ;(\{s-u, t-u\}, t-\tilde{u}, t-u, t-\tilde{u})) .
$$

In the sequel, and for the sake of clarity, we will only focus on the estimates associated with this particular pair $(a ; b)$, but it should (again) be clear to the reader that any other pair $(a ; b)$ satisfying the above conditions could be treated with similar arguments.

Injecting successively (51), (52) and (53) into (50) (with $(a ; b)$ fixed as in (54)) gives rise to the consideration of three specific integrals, that we denote by $\mathcal{J}_{1}, \mathcal{J}_{2}$ and $\mathcal{J}_{3}$, respectively.

Estimation of $\mathcal{J}_{1}$. Using the covariance formula (34), we get on the one hand

$$
\begin{aligned}
& \iint_{\left(\mathbb{R}^{2}\right)^{2}} d y d \tilde{y} e^{-\imath\langle\beta, y\rangle} e^{\imath\langle\tilde{\beta}, \tilde{y}\rangle} \mathbb{E}\left[\varphi_{s-u, t-u}^{n, m}(y) \overline{?_{t-\tilde{u}}^{m}(\tilde{y})}\right] \mathbb{E}\left[?_{t-u}^{m}(y) \overline{\varphi_{t-\tilde{u}}^{m}(\tilde{y})}\right] \\
& =c \iint_{\left.\mathbb{R}^{2}\right)^{2}} d \eta d \tilde{\eta} L_{(s-u, t-u), t-\tilde{u}}^{H,((n, m), m)}(\eta) L_{t-u, t-\tilde{u}}^{H,(m, m)}(\tilde{\eta})\left(\int_{\mathbb{R}^{2}} d y e^{-\imath\langle y, \beta-(\eta+\tilde{\eta})\rangle}\right)\left(\int_{\mathbb{R}^{2}} d \tilde{y} e^{\imath\langle\tilde{y}, \tilde{\beta}-(\eta+\tilde{\eta})\rangle}\right) \\
& =c \iint_{\left(\mathbb{R}^{2}\right)^{2}} d \eta d \tilde{\eta} L_{(s-u, t-u), t-\tilde{u}}^{H,((n, m), m)}(\eta) L_{t-u, t-\tilde{u}}^{H,(m, m)}(\tilde{\eta}) \delta_{\{\beta=\tilde{\beta}=\eta+\tilde{\eta}\}},
\end{aligned}
$$

and on the other hand

$$
\iint_{\left(\mathbb{R}^{2}\right)^{2}} d z d \tilde{z} e^{-\imath\langle\lambda-\beta, z\rangle} e^{\imath\langle\tilde{\lambda}-\tilde{\beta}, \tilde{z}\rangle} \mathbb{E}\left[\varrho_{\bullet t}^{m}(z) \overline{\varrho_{t}^{m}(\tilde{z})}\right]=c \int_{\mathbb{R}^{2}} d \tilde{\tilde{\eta}} L_{t, t}^{H,(m, m)}(\tilde{\tilde{\eta}}) \delta_{\{\lambda-\beta=\tilde{\lambda}-\tilde{\beta}=\tilde{\tilde{\eta}}\}}
$$


so that

$$
\begin{gathered}
\mathcal{J}_{1} \lesssim \iint_{[0, t]^{2}} d u d \tilde{u} \iiint_{\left(\mathbb{R}^{2}\right)^{3}} \frac{d \eta d \tilde{\eta} d \tilde{\tilde{\eta}}}{\left\{1+|\eta+\tilde{\eta}+\tilde{\tilde{\eta}}|^{2}\right\}^{\alpha}}\left|L_{(s-u, t-u), t-\tilde{u}}^{H,((n, m), m)}(\eta)\right| L_{t-u, t-\tilde{u}}^{H,(m, m)}(\tilde{\eta})|| L_{t, t}^{H,(m, m)}(\tilde{\tilde{\eta}}) \mid \\
\lesssim \iint_{[0, t]^{2}} d u d \tilde{u} \iint_{\left(\mathbb{R}^{2}\right)^{2}} d \eta d \tilde{\tilde{\eta}}\left\{1+|\eta+\tilde{\tilde{\eta}}|^{2}\right\}^{-\alpha}\left|\mathcal{F}_{x}\left(G_{u}\right)(\eta)\right|\left|\mathcal{F}_{x}\left(G_{\tilde{u}}\right)(\eta)\right| \\
\left|L_{t, t}^{H,(m, m)}(\tilde{\tilde{\eta}})\right|\left(\int_{\mathbb{R}^{2}} d \tilde{\eta}\left|L_{(s-u, t-u), t-\tilde{u}}^{H,((n, m), m)}(\eta-\tilde{\eta})\right|\left|L_{t-u, t-\tilde{u}}^{H,(m, m)}(\tilde{\eta})\right|\right) .
\end{gathered}
$$

At this point, we can apply Lemma 3.1 to assert that for any $\varepsilon>0$ small enough,

$$
\begin{aligned}
& \left|L_{t, t}^{H,(m, m)}(\tilde{\tilde{\eta}})\right|\left(\int_{\mathbb{R}^{2}} d \tilde{\eta}\left|L_{(s-u, t-u), t-\tilde{u}}^{H,((n, m), m)}(\eta-\tilde{\eta})\right|\left|L_{t-u, t-\tilde{u}}^{H,(m, m)}(\tilde{\eta})\right|\right) \\
& \lesssim 2^{-n \varepsilon}|t-s|^{\varepsilon} K^{H_{\varepsilon, 0}}(\tilde{\tilde{\eta}}) \sum_{i=0}^{2}\left(\int_{\mathbb{R}^{2}} d \tilde{\eta} K^{H_{\varepsilon, i}}(\eta-\tilde{\eta}) K^{H_{\varepsilon, 0}}(\tilde{\eta})\right) .
\end{aligned}
$$

Thanks to the forthcoming Lemma 3.4, we know that for every $i \in\{0,1,2\}$ and for any $\varepsilon>0$ small enough,

$$
\sup _{\eta \in \mathbb{R}^{2}} \int_{\mathbb{R}^{2}} d \tilde{\eta} K^{H_{\varepsilon, i}}(\eta-\tilde{\eta}) K^{H_{\varepsilon, 0}}(\tilde{\eta}) \leq\left(\int_{\mathbb{R}^{2}} d \tilde{\eta}\left|K^{H_{\varepsilon, i}}(\tilde{\eta})\right|^{2}\right)^{\frac{1}{2}}\left(\int d \tilde{\eta}\left|K^{H_{\varepsilon, 0}}(\tilde{\eta})\right|^{2}\right)^{\frac{1}{2}}<\infty .
$$

Going back to (57) and using also (49), we have thus obtained that

$$
\mathcal{J}_{1} \lesssim 2^{-n \varepsilon}|t-s|^{\varepsilon} \int_{\mathbb{R}^{2}} d \tilde{\tilde{\eta}} K^{H_{\varepsilon, 0}}(\tilde{\tilde{\eta}}) \int_{\mathbb{R}^{2}} d \eta\left\{1+|\eta+\tilde{\tilde{\eta}}|^{2}\right\}^{-\alpha}\left\{1+|\eta|^{2}\right\}^{-1}
$$

Applying the subsequent technical Lemma 3.5 finally yields

$$
\mathcal{J}_{1} \lesssim 2^{-n \varepsilon}|t-s|^{\varepsilon} \int_{\mathbb{R}^{2}} \frac{d \tilde{\tilde{\eta}}}{\left\{1+|\tilde{\tilde{\eta}}|^{2}\right\}^{\alpha-\varepsilon}} K^{H_{\varepsilon, 0}}(\tilde{\tilde{\eta}})
$$

and the conclusion now comes from the first-order assertion (40).

Estimation of $\mathcal{J}_{2}$. Using the same arguments as in (55) (with the help of both (34) and (35)), we get

$$
\begin{aligned}
& \iint_{\left(\mathbb{R}^{2}\right)^{2}} d y d \tilde{y} \iint_{\left(\mathbb{R}^{2}\right)^{2}} d z d \tilde{z} e^{-\imath\langle\beta, y\rangle} e^{\imath\langle\tilde{\beta}, \tilde{y}\rangle} e^{-\imath\langle\lambda-\beta, z\rangle} e^{\imath\langle\tilde{\lambda}-\tilde{\beta}, \tilde{z}\rangle} \\
& \mathbb{E}\left[\varphi_{s-u, t-u}^{n, m}(y) \overline{?_{t-\tilde{u}}^{m}(\tilde{y})}\right] \mathbb{E}\left[?_{t-u}^{m}(y) ?_{t}^{m}(z)\right] \mathbb{E}\left[\overline{?_{t-\tilde{u}}^{m}(\tilde{y}) ?_{t}^{m}(\tilde{z})}\right] \\
& =c \iiint_{\left(\mathbb{R}^{2}\right)^{3}} d \eta d \tilde{\eta} d \tilde{\tilde{\eta}} L_{(s-u, t-u), t-\tilde{u}}^{H,((n), m), m)}\left(\eta \tilde{L}_{t-u, t}^{H,(m, m)}(\tilde{\eta}) \overline{\tilde{L}_{t-\tilde{u}, t}^{H,(m, m)}(\tilde{\tilde{\eta}})} \delta_{\{\beta=\eta+\tilde{\eta}\}} \delta_{\{\tilde{\beta}=\eta+\tilde{\tilde{\eta}}\}} \delta_{\{\lambda=\eta+2 \tilde{\eta}\}} \delta_{\{\tilde{\lambda}=\eta+2 \tilde{\tilde{\eta}}\}},\right.
\end{aligned}
$$

and so

$$
\begin{gathered}
\mathcal{J}_{2} \lesssim \iint_{[0, t]^{2}} d u d \tilde{u} \iiint_{\left(\mathbb{R}^{2}\right)^{3}} d \eta d \tilde{\eta} d \tilde{\tilde{\eta}}\left|L_{(s-u, t-u), t-\tilde{u}}^{H,((n, m), m)}(\eta)\right|\left|\tilde{L}_{t-u, t}^{H,(m, m)}(\tilde{\eta})\right|\left|\tilde{L}_{t-\tilde{u}, t}^{H,(m, m)}(\tilde{\tilde{\eta}})\right| \\
\left\{1+|\eta+2 \tilde{\eta}|^{2}\right\}^{-\frac{\alpha}{2}}\left\{1+|\eta+2 \tilde{\tilde{\eta}}|^{2}\right\}^{-\frac{\alpha}{2}}\left|\mathcal{F}_{x}\left(G_{u}\right)(\eta+\tilde{\eta})\right|\left|\mathcal{F}_{x}\left(G_{\tilde{u}}\right)(\eta+\tilde{\tilde{\eta}})\right| \\
\lesssim \iint_{[0, t]^{2}} d u d \tilde{u} \int_{\mathbb{R}^{2}} d \eta\left|L_{(s-u, t-u), t-\tilde{u}}^{H,((n, m), m)}(\eta)\right|\left(\int_{\mathbb{R}^{2}} d \tilde{\eta}\left|\tilde{L}_{t-u, t}^{H,(m, m)}(\tilde{\eta})\right|\left\{1+|\eta+2 \tilde{\eta}|^{2}\right\}^{-\frac{\alpha}{2}}\left|\mathcal{F}_{x}\left(G_{u}\right)(\eta+\tilde{\eta})\right|\right) \\
\left(\int_{\mathbb{R}^{2}} d \tilde{\tilde{\eta}}\left|\tilde{L}_{t-\tilde{u}, t}^{H,(m, m)}(\tilde{\tilde{\eta}})\right|\left\{1+|\eta+2 \tilde{\tilde{\eta}}|^{2}\right\}^{-\frac{\alpha}{2}}\left|\mathcal{F}_{x}\left(G_{\tilde{u}}\right)(\eta+\tilde{\eta})\right|\right)
\end{gathered}
$$


Combining (36) with the result of Lemma 3.4 and estimate (49), we can assert that for any $\varepsilon>0$ small enough,

$$
\begin{aligned}
& \sup _{u \in[0, t]}\left|\int_{\mathbb{R}^{2}} d \tilde{\eta}\right| \tilde{L}_{t-u, t}^{H,(m, m)}(\tilde{\eta})\left|\left\{1+|\eta+2 \tilde{\eta}|^{2}\right\}^{-\frac{\alpha}{2}}\right| \mathcal{F}_{x}\left(G_{u}\right)(\eta+\tilde{\eta})|| \\
& \quad \lesssim\left(\int_{\mathbb{R}^{2}} d \tilde{\eta}\left|K^{H_{\varepsilon, 0}}(\tilde{\eta})\right|^{2}\right)^{1 / 2}\left(\int_{\mathbb{R}^{2}} d \tilde{\eta}\left\{1+|\eta+2 \tilde{\eta}|^{2}\right\}^{-\alpha}\left\{1+|\eta+\tilde{\eta}|^{2}\right\}^{-1}\right)^{1 / 2} \\
& \quad \lesssim\left(\int_{\mathbb{R}^{2}} d \tilde{\eta}\left\{1+|\eta+\tilde{\eta}|^{2}\right\}^{-\alpha}\left\{1+|\tilde{\eta}|^{2}\right\}^{-1}\right)^{1 / 2}
\end{aligned}
$$

and similarly

$$
\sup _{\tilde{u} \in[0, t]}\left|\int_{\mathbb{R}^{2}} d \tilde{\tilde{\eta}}\right| \tilde{L}_{t-\tilde{u}, t}^{H,(m, m)}(\tilde{\tilde{\eta}})\left|\left\{1+|\eta+2 \tilde{\tilde{\eta}}|^{2}\right\}^{-\frac{\alpha}{2}}\right| \mathcal{F}_{x}\left(G_{t-\tilde{u}}\right)(\eta+\tilde{\tilde{\eta}})|| \lesssim\left(\int_{\mathbb{R}^{2}} d \tilde{\tilde{\eta}}\left\{1+|\eta+\tilde{\tilde{\eta}}|^{2}\right\}^{-\alpha}\left\{1+|\tilde{\tilde{\eta}}|^{2}\right\}^{-1}\right)^{1 / 2},
$$

which, going back to (59), yields

$$
\begin{aligned}
\mathcal{J}_{2} & \lesssim \iint_{[0, t]^{2}} d u d \tilde{u} \int_{\mathbb{R}^{2}} d \eta \mid L_{(s-u, t-u), t-\tilde{u}}^{H,((n, m), m)}(\eta) \int_{\mathbb{R}^{2}} d \tilde{\eta}\left\{1+|\eta+\tilde{\eta}|^{2}\right\}^{-\alpha}\left\{1+|\tilde{\eta}|^{2}\right\}^{-1} \\
& \lesssim 2^{-n \varepsilon}|t-s|^{\varepsilon} \sum_{i=0}^{2} \int_{\mathbb{R}^{2}} d \eta K^{H_{\varepsilon, i}}(\eta) \int_{\mathbb{R}^{2}} d \tilde{\eta}\left\{1+|\eta+\tilde{\eta}|^{2}\right\}^{-\alpha}\left\{1+|\tilde{\eta}|^{2}\right\}^{-1} .
\end{aligned}
$$

We can then conclude with the same arguments as in (58).

Estimation of $\mathcal{J}_{3}$. As above,

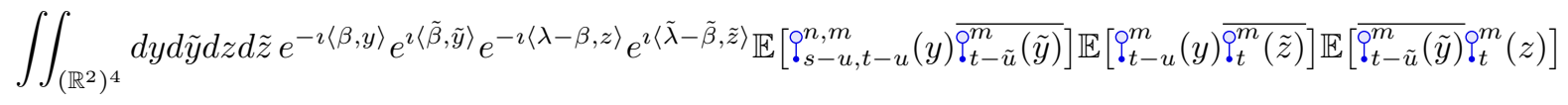

$$
\begin{aligned}
& =c \iiint_{\left(\mathbb{R}^{2}\right)^{3}} d \eta d \tilde{\eta} d \tilde{\tilde{\eta}} L_{(s-u, t-u), t-\tilde{u}}^{H,((n, m), m)}(\eta) L_{t-u, t}^{H,(m, m)}(\tilde{\eta}) \overline{L_{t-\tilde{u}, t}^{H,(m, m)}(\tilde{\tilde{\eta}})} \delta_{\{\beta=\eta+\tilde{\eta}\}} \delta_{\{\tilde{\beta}=\eta+\tilde{\tilde{\eta}}\}} \delta_{\{\lambda=\tilde{\lambda}=\eta+\tilde{\eta}+\tilde{\eta}\}},
\end{aligned}
$$

and thus, for any $\varepsilon>0$ small enough,

$$
\begin{gathered}
\mathcal{J}_{3} \lesssim \iint_{[0, t]^{2}} d u d \tilde{u} \iiint_{\left(\mathbb{R}^{2}\right)^{3}} \frac{d \eta d \tilde{\eta} d \tilde{\tilde{\eta}}}{\left\{1+|\eta+\tilde{\eta}+\tilde{\tilde{\eta}}|^{2}\right\}^{\alpha}} \mid L_{(s-u, t-u), t-\tilde{u}}^{H,((n), m)|| L_{t-u, t}^{H,(m, m)}(\tilde{\eta})|| L_{t-\tilde{u}, t}^{H,(m, m)}(\tilde{\tilde{\eta}}) \mid} \\
\left|\mathcal{F}_{x}\left(G_{u}\right)(\eta+\tilde{\eta})\right|\left|\mathcal{F}_{x}\left(G_{\tilde{u}}\right)(\eta+\tilde{\tilde{\eta}})\right| \\
\lesssim 2^{-n \varepsilon}|t-s|^{\varepsilon} \sum_{i=0}^{2} \iiint_{\left(\mathbb{R}^{2}\right)^{3}} d \eta d \tilde{\eta} d \tilde{\tilde{\eta}} K^{H_{\varepsilon, i}}(\eta) K^{H_{\varepsilon, 0}}(\tilde{\eta}) K^{H_{\varepsilon, 0}}(\tilde{\tilde{\eta}}) \\
\left\{1+|\eta+\tilde{\eta}+\tilde{\tilde{\eta}}|^{2}\right\}^{-\alpha}\left\{1+|\eta+\tilde{\eta}|^{2}\right\}^{-\frac{1}{2}}\left\{1+|\eta+\tilde{\tilde{\eta}}|^{2}\right\}^{-\frac{1}{2}} .
\end{gathered}
$$

Now split the integration domain into $\mathcal{D}_{1} \triangleq\left\{(\eta, \tilde{\eta}, \tilde{\tilde{\eta}}):\left\{1+|\eta+\tilde{\eta}|^{2}\right\}^{-\frac{1}{2}} \leq\left\{1+|\eta+\tilde{\tilde{\eta}}|^{2}\right\}^{-\frac{1}{2}}\right\}$ and $\mathcal{D}_{2} \triangleq$ $\left\{(\eta, \tilde{\eta}, \tilde{\tilde{\eta}}):\left\{1+|\eta+\tilde{\tilde{\eta}}|^{2}\right\}^{-\frac{1}{2}} \leq\left\{1+|\eta+\tilde{\eta}|^{2}\right\}^{-\frac{1}{2}}\right\}$, and write (trivially)

$$
\begin{aligned}
& \iiint_{\mathcal{D}_{1}} d \eta d \tilde{\eta} d \tilde{\tilde{\eta}} K^{H_{\varepsilon, i}}(\eta) K^{H_{\varepsilon, 0}}(\tilde{\eta}) K^{H_{\varepsilon, 0}}(\tilde{\tilde{\eta}})\left\{1+|\eta+\tilde{\eta}+\tilde{\tilde{\eta}}|^{2}\right\}^{-\alpha}\left\{1+|\eta+\tilde{\eta}|^{2}\right\}^{-\frac{1}{2}}\left\{1+|\eta+\tilde{\tilde{\eta}}|^{2}\right\}^{-\frac{1}{2}} \\
& \leq \iiint_{\left(\mathbb{R}^{2}\right)^{3}} d \eta d \tilde{\eta} d \tilde{\tilde{\eta}} K^{H_{\varepsilon, i}}(\eta) K^{H_{\varepsilon, 0}}(\tilde{\eta}) K^{H_{\varepsilon, 0}}(\tilde{\tilde{\eta}})\left\{1+|\eta+\tilde{\eta}+\tilde{\tilde{\eta}}|^{2}\right\}^{-\alpha}\left\{1+|\eta+\tilde{\tilde{\eta}}|^{2}\right\}^{-1},
\end{aligned}
$$

which essentially brings us back to the integral involved in (56). We can thus rely on the same arguments as with $\mathcal{J}_{1}$ to handle the integral over $\mathcal{D}_{1}$. Finally, it is readily checked that these arguments can also be used for the integral over $\mathcal{D}_{2}$, which concludes the proof.

It only remains us to prove the two technical lemmas at the core of the above reasoning. 
Lemma 3.4. For all $\left(H_{0}, H_{1}, H_{2}\right) \in(0,1)^{3}$ such that

$$
0<H_{1}<\frac{3}{4} \quad, \quad 0<H_{2}<\frac{3}{4} \quad \text { and } \quad H_{0}+H_{1}+H_{2}>1,
$$

it holds that

$$
\int_{\mathbb{R}^{2}} d \eta\left|K^{H}(\eta)\right|^{2}<\infty
$$

Proof. One has, since $4 H_{1}-2<1$ and $4 H_{2}-2<1$,

$$
\int_{\mathbb{R}^{2}} d \eta\left|K^{H}(\eta)\right|^{2} \lesssim \iint_{\mathbb{R}^{2}} \frac{d \eta_{1} d \eta_{2}}{\left|\eta_{1}\right|^{4 H_{1}-2}\left|\eta_{2}\right|^{4 H_{2}-2}\left\{1+|\eta|^{2+4 H_{0}}\right\}} \lesssim \int_{0}^{\infty} \frac{d \rho}{\rho^{4\left(H_{1}+H_{2}\right)-5}\left\{1+\rho^{\left.2+4 H_{0}\right\}}\right.},
$$

and we can easily check (using (60)) that the latter integral is indeed finite.

Lemma 3.5. For all $0<\varepsilon<\alpha<\frac{1}{2}$, it holds that

$$
\int_{\mathbb{R}^{2}} d \tilde{\eta}\left\{1+|\eta+\tilde{\eta}|^{2}\right\}^{-\alpha}\left\{1+|\tilde{\eta}|^{2}\right\}^{-1} \lesssim\left\{1+|\eta|^{2}\right\}^{-(\alpha-\varepsilon)} .
$$

Proof. Let us first write

$\int_{\mathbb{R}^{2}} d \tilde{\eta}\left\{1+|\eta+\tilde{\eta}|^{2}\right\}^{-\alpha}\left\{1+|\tilde{\eta}|^{2}\right\}^{-1} \lesssim \int_{\mathbb{R}^{2}} d \tilde{\eta}\left\{1+|| \eta|-| \tilde{\eta}||^{2}\right\}^{-\alpha}\left\{1+|\tilde{\eta}|^{2}\right\}^{-1} \lesssim \int_{0}^{\infty} \frac{d \tau \tau}{1+\tau^{2}}\left\{1+|| \eta|-\tau|^{2}\right\}^{-\alpha}$.

Now split the integration domain into $\mathcal{D}_{1} \triangleq\left[\frac{|\eta|}{2}, \frac{3|\eta|}{2}\right]$ and $\mathcal{D}_{2} \triangleq\left\{0 \leq \tau \leq \frac{|\eta|}{2}\right.$ or $\left.\tau \geq \frac{3|\eta|}{2}\right\}$. On the one hand,

$$
\begin{aligned}
\int_{\mathcal{D}_{1}} \frac{d \tau \tau}{1+\tau^{2}}\left\{1+|| \eta|-\tau|^{2}\right\}^{-\alpha} & =|\eta|^{2} \int_{\frac{1}{2}}^{\frac{3}{2}} \frac{d r r}{\left\{1+|\eta|^{2}(1-r)^{2}\right\}^{\alpha}\left\{1+|\eta|^{2} r^{2}\right\}} \\
& \lesssim \int_{-\frac{1}{2}}^{\frac{1}{2}} \frac{d r}{\left\{1+|\eta|^{2} r^{2}\right\}^{\alpha}} \lesssim \max \left(1, \frac{1}{|\eta|^{2 \alpha}} \int_{0}^{1} \frac{d r}{r^{2 \alpha}}\right) \lesssim \frac{1}{1+|\eta|^{2 \alpha}}
\end{aligned}
$$

On the other hand, for every $\tau \in \mathcal{D}_{2}$, one has ||$\eta|-\tau| \geq \frac{1}{3} \max (\tau,|\eta|)$, and accordingly

$$
\int_{\mathcal{D}_{2}} \frac{d \tau \tau}{1+\tau^{2}}\left\{1+|| \eta|-\tau|^{2}\right\}^{-\alpha} \lesssim\left\{1+|\eta|^{2}\right\}^{-(\alpha-\varepsilon)} \int_{0}^{\infty} \frac{d \tau \tau}{\left\{1+\tau^{2}\right\}^{1+\varepsilon}} \lesssim\left\{1+|\eta|^{2}\right\}^{-(\alpha-\varepsilon)} .
$$

\subsection{Proof of Proposition 1.4}

In the sequel, we use the notation $A \gtrsim B$ whenever there exists a constant $c>0$ such that $A \geq c B$. Besides, without loss of generality, we can here assume that $\alpha>\frac{1}{2}$. For the sake of clarity, let us also introduce the additional notation

$$
\Gamma_{t}^{H_{0}, n}(\tau) \triangleq \int_{-2^{n}}^{2^{n}} d \xi \frac{\left|\gamma_{t}(\xi, \tau)\right|^{2}}{|\xi|^{2 H_{0}-1}}
$$

Using (34) and then Wick formula just as in Section 3.1.2, we get that

$$
\begin{aligned}
\mathbb{E} & {\left[\left\|\rho \cdot Q \rho^{n}(t, .)\right\|_{\mathcal{W}^{-2 \alpha, 2}}^{2}\right] } \\
= & c \int_{|\eta| \leq 2^{n}} d \eta \int_{|\tilde{\eta}| \leq 2^{n}} d \tilde{\eta} \frac{\Gamma_{t}^{H_{0}, n}(|\eta|)}{\left|\eta_{1}\right|^{2 H_{1}-1}\left|\eta_{2}\right|^{2 H_{2}-1}} \frac{\Gamma_{t}^{H_{0}, n}(|\tilde{\eta}|)}{\left|\tilde{\eta}_{1}\right|^{2 H_{1}-1}\left|\tilde{\eta}_{2}\right|^{2 H_{2}-1}} \int_{\mathbb{R}^{2}} \frac{d \xi}{\left\{1+|\xi|^{2}\right\}^{2 \alpha}}|\widehat{\rho}(\xi-(\eta-\tilde{\eta}))|^{2} . \\
& \text { imsart-aihp ver. 2014/10/16 file: quadratic-wave-rougher-accepted-version.tex date: February 14, } 2019
\end{aligned}
$$


Then observe that

$$
\begin{aligned}
& \int_{\mathbb{R}^{2}} \frac{d \xi}{\left\{1+|\xi|^{2}\right\}^{2 \alpha}}|\widehat{\rho}(\xi-(\eta-\tilde{\eta}))|^{2} \\
& =\int_{\mathbb{R}^{2}} \frac{d \xi}{\left\{1+|\xi+(\eta-\tilde{\eta})|^{2}\right\}^{2 \alpha}}|\widehat{\rho}(\xi)|^{2} \gtrsim \frac{1}{\left\{1+|\eta-\tilde{\eta}|^{2}\right\}^{2 \alpha}} \int_{\mathbb{R}^{2}} \frac{d \xi}{\left\{1+|\xi|^{2}\right\}^{2 \alpha}}|\widehat{\rho}(\xi)|^{2},
\end{aligned}
$$

and so

$$
\begin{aligned}
& \mathbb{E}\left[\left\|\rho \cdot Q^{n} \rho^{n}(t, .)\right\|_{\mathcal{W}-2 \alpha, 2}^{2}\right] \\
& \gtrsim \int_{0}^{2^{n-1}} d \eta_{1} \int_{\frac{1}{2} \eta_{1}}^{\eta_{1}} d \tilde{\eta}_{1} \int_{0}^{2^{n-1}} d \eta_{2} \int_{\frac{1}{2} \eta_{2}}^{\eta_{2}} d \tilde{\eta}_{2} \frac{1}{\left\{1+|\eta-\tilde{\eta}|^{2}\right\}^{2 \alpha}} \frac{\Gamma_{t}^{H_{0}, n}(|\eta|)}{\left|\eta_{1}\right|^{2 H_{1}-1}\left|\eta_{2}\right|^{2 H_{2}-1}} \frac{\Gamma_{t}^{H_{0}, n}(|\tilde{\eta}|)}{\left|\tilde{\eta}_{1}\right|^{2 H_{1}-1}\left|\tilde{\eta}_{2}\right|^{2 H_{2}-1}} \\
& \gtrsim \int_{0}^{2^{n-1}} \int_{0}^{2^{n-1}} \frac{d \eta_{1} d \eta_{2}}{\left|\eta_{1}\right|^{4 H_{1}-3}\left|\eta_{2}\right|^{4 H_{2}-3}} \int_{0}^{\frac{1}{2}} \int_{0}^{\frac{1}{2}} \frac{d r_{1} d r_{2}}{\left\{1+\eta_{1}^{2} r_{1}^{2}+\eta_{2}^{2} r_{2}^{2}\right\}^{2 \alpha}} \\
& \Gamma_{t}^{H_{0}, n}(|\eta|) \Gamma_{t}^{H_{0}, n}\left(\sqrt{\eta_{1}^{2}\left(1-r_{1}\right)^{2}+\eta_{2}^{2}\left(1-r_{2}\right)^{2}}\right) \\
& \gtrsim \int_{0<\eta_{1}<\eta_{2}<2^{n-1}} \frac{d \eta_{1} d \eta_{2}}{\left|\eta_{1}\right|^{4 H_{1}-2}\left|\eta_{2}\right|^{4 H_{2}-2}} \int_{0}^{\frac{1}{2} \eta_{1}} \int_{0}^{\frac{1}{2} \eta_{1}} \frac{d r_{1} d r_{2}}{\left\{1+r_{1}^{2}+r_{2}^{2}\right\}^{2 \alpha}} \\
& \Gamma_{t}^{H_{0}, n}(|\eta|) \Gamma_{t}^{H_{0}, n}\left(\sqrt{\eta_{1}^{2}\left(1-\frac{r_{1}}{\eta_{1}}\right)^{2}+\eta_{2}^{2}\left(1-\frac{r_{2}}{\eta_{2}}\right)^{2}}\right) \\
& \gtrsim \int_{\frac{\pi}{8}}^{\frac{\pi}{4}} d \theta \int_{2}^{2^{n-1}} \frac{d \tau}{\tau^{4 H_{1}^{\prime}+4 H_{2}^{\prime}-5}} \int_{0}^{\frac{1}{2} \tau \sin \theta} \int_{0}^{\frac{1}{2} \tau \sin \theta} \frac{d r_{1} d r_{2}}{\left\{1+r_{1}^{2}+r_{2}^{2}\right\}^{2 \alpha}} \\
& \Gamma_{t}^{H_{0}, n}(\tau) \Gamma_{t}^{H_{0}, n}\left(\sqrt{\tau^{2} \sin ^{2} \theta\left(1-\frac{r_{1}}{\tau \sin \theta}\right)^{2}+\tau^{2} \cos ^{2} \theta\left(1-\frac{r_{2}}{\tau \cos \theta}\right)^{2}}\right),
\end{aligned}
$$

where, for technical reasons (in subsequent arguments), we have picked $H_{1}^{\prime} \geq H_{1}$ and $H_{2}^{\prime} \geq H_{2}$ such that $\frac{3}{4}<H_{0}+H_{1}^{\prime}+H_{2}^{\prime} \leq 1$. At this point, observe that for all $\theta \in\left(\frac{\pi}{8}, \frac{\pi}{4}\right), \tau \in\left(2,2^{n-1}\right)$ and $r_{1}, r_{2} \in\left(0, \frac{1}{2} \tau \sin \theta\right)$, we have

$$
\tau \geq \tau_{r, \theta} \triangleq \sqrt{\tau^{2} \sin ^{2} \theta\left(1-\frac{r_{1}}{\tau \sin \theta}\right)^{2}+\tau^{2} \cos ^{2} \theta\left(1-\frac{r_{2}}{\tau \cos \theta}\right)^{2}} \geq \frac{1}{2} \tau \geq 1 .
$$

We are therefore in a position to apply the (forthcoming) lower bound (63), which entails, with the notation of Lemma 3.6,

$$
\begin{gathered}
\mathbb{E}\left[\left\|\rho \cdot \rho_{0} \rho^{n}(t, .)\right\|_{\mathcal{W}^{-2 \alpha, 2}}^{2}\right] \gtrsim \int_{\frac{\pi}{8}}^{\frac{\pi}{4}} d \theta \int_{2}^{2^{n-1}} \frac{d \tau}{\tau^{4 H_{1}^{\prime}+4 H_{2}^{\prime}-5}} \\
\int_{0}^{\frac{1}{2} \tau \sin \theta} \int_{0}^{\frac{1}{2} \tau \sin \theta} \frac{d r_{1} d r_{2}}{\left\{1+r_{1}^{2}+r_{2}^{2}\right\}^{2 \alpha}}\left[\frac{c t}{\tau^{1+2 H_{0}}}+Q_{t}^{H_{0}}(\tau)\right]\left[\frac{c t}{\tau^{1+2 H_{0}}}+Q_{t}^{H_{0}}\left(\tau_{r, \theta}\right)\right] \\
\gtrsim t^{2}\left(\int_{0}^{\sin \frac{\pi}{8}} \int_{0}^{\sin \frac{\pi}{8}} \frac{d r_{1} d r_{2}}{\left\{1+r_{1}^{2}+r_{2}^{2}\right\}^{2 \alpha}}\right)\left(\int_{2}^{2^{n-1}} \frac{d \tau}{\tau^{4\left(H_{0}+H_{1}^{\prime}+H_{2}^{\prime}\right)-3}}\right)+R_{t}^{n}
\end{gathered}
$$

where we have set

$$
\begin{aligned}
R_{t}^{n} \triangleq & \int_{\frac{\pi}{8}}^{\frac{\pi}{4}} d \theta \int_{2}^{2^{n-1}} \frac{d \tau}{\tau^{4 H_{1}^{\prime}+4 H_{2}^{\prime}-5}} \\
& \int_{0}^{\frac{1}{2} \tau \sin \theta} \int_{0}^{\frac{1}{2} \tau \sin \theta} \frac{d r_{1} d r_{2}}{\left\{1+r_{1}^{2}+r_{2}^{2}\right\}^{2 \alpha}}\left[\frac{c t}{\tau^{1+2 H_{0}}} Q_{H_{0}}\left(\tau_{r, \theta}\right)+\frac{c t}{\tau^{1+2 H_{0}}} Q_{H_{0}}(\tau)+Q_{H_{0}}(\tau) Q_{H_{0}}\left(\tau_{r, \theta}\right)\right] .
\end{aligned}
$$

Let us now show that $\left|R_{t}^{n}\right|$ is uniformly bounded with respect to $n$. In fact, thanks to (64), we can assert 
that for any $\varepsilon>0$,

$$
\sup _{t \in[0,1]}\left|\frac{c}{\tau^{1+2 H_{0}}} Q_{t}^{H_{0}}\left(\tau_{r, \theta}\right)+\frac{c}{\tau^{1+2 H_{0}}} Q_{t}^{H_{0}}(\tau)+Q_{t}^{H_{0}}(\tau) Q_{t}^{H_{0}}\left(\tau_{r, \theta}\right)\right| \lesssim \frac{1}{\tau^{3+4 H_{0}-\varepsilon}} .
$$

Therefore,

$$
\begin{aligned}
\sup _{t \in[0,1]}\left|R_{t}^{n}\right| & \lesssim\left(\int_{0}^{\infty} \int_{0}^{\infty} \frac{d r_{1} d r_{2}}{\left\{1+r_{1}^{2}+r_{2}^{2}\right\}^{2 \alpha}}\right)\left(\int_{2}^{\infty} \frac{d \tau}{\tau^{4\left(H_{0}+H_{1}^{\prime}+H_{2}^{\prime}\right)-2-\varepsilon}}\right) \\
& \lesssim\left(\int_{0}^{\infty} d r \frac{r}{\left\{1+r^{2}\right\}^{2 \alpha}}\right)\left(\int_{2}^{\infty} \frac{d \tau}{\tau^{4\left(H_{0}+H_{1}^{\prime}+H_{2}^{\prime}\right)-2-\varepsilon}}\right),
\end{aligned}
$$

and, provided $\varepsilon>0$ is picked small enough, these two integrals are obviously finite, due to $\alpha>\frac{1}{2}$ and $H_{0}+H_{1}^{\prime}+H_{2}^{\prime}>\frac{3}{4}$.

Going back to (62), we get the conclusion since, as $H_{0}+H_{1}^{\prime}+H_{2}^{\prime} \leq 1$,

$$
\int_{2}^{2^{n-1}} \frac{d \tau}{\tau^{4\left(H_{0}+H_{1}^{\prime}+H_{2}^{\prime}\right)-3}} \stackrel{n \rightarrow \infty}{\longrightarrow} \infty
$$

Lemma 3.6. There exists a constant $c>0$ such that for all $H_{0} \in(0,1), \varepsilon>0, n \geq 1, t \in[0,1]$ and $\tau \in\left(1,2^{n}\right)$,

$$
\Gamma_{t}^{H_{0}, n}(\tau) \geq \frac{c t}{\tau^{1+2 H_{0}}}+Q_{t}^{H_{0}}(\tau) \geq 0,
$$

with $Q^{H_{0}}$ satisfying

$$
\sup _{t \in[0,1]}\left|Q_{t}^{H_{0}}(\tau)\right| \leq \frac{c_{\varepsilon, H_{0}}}{\tau^{2+2 H_{0}-\varepsilon}} .
$$

Proof. We will lean on similar estimates as those of the proof of [1, Proposition 2.4]. Let us first recall the explicit expression (see the latter reference) $\left|\gamma_{t}(\xi, \tau)\right|^{2}=c\left\{\Lambda_{t}(\xi, \tau)+\Lambda_{t}(-\xi, \tau)\right\}$, with $c>0$ and

$$
\Lambda_{t}(\xi, \tau) \triangleq \frac{1-\cos (t(\xi-\tau))}{\tau^{2}(\xi-\tau)^{2}}+\frac{\cos (t \tau)\{\cos (t \xi)-\cos (t \tau)\}}{\tau^{2}(\xi-\tau)(\xi+\tau)} .
$$

Thus, one has, for any $\tau \in\left(1,2^{n}\right)$,

$$
\Gamma_{t}^{H_{0}, n}(\tau) \geq \int_{-\tau}^{\tau} d \xi \frac{\left|\gamma_{t}(\xi, \tau)\right|^{2}}{|\xi|^{2 H_{0}-1}}=2 c \int_{-\tau}^{\tau} d \xi \frac{\Lambda_{t}(\xi, \tau)}{|\xi|^{2 H_{0}-1}} \geq 0
$$

Decompose $\Lambda_{t}(\xi, \tau) \mathbf{1}_{\{-\tau<\xi<\tau\}}$ into $\Lambda_{t}(\xi, \tau) \mathbf{1}_{\{-\tau<\xi<\tau\}}=\Lambda_{t}^{1}(\xi, \tau)+\Lambda_{t}^{2}(\xi, \tau)$, with

$$
\Lambda_{t}^{1}(\xi, \tau) \triangleq \frac{1-\cos (t(\xi-\tau))}{\tau^{2}(\xi-\tau)^{2}} \mathbf{1}_{\left\{\xi \geq \frac{\tau}{2}\right\}}
$$

and

$$
\Lambda_{t}^{2}(\xi, \tau) \triangleq \frac{1-\cos (t(\xi-\tau))}{\tau^{2}(\xi-\tau)^{2}} \mathbf{1}_{\left\{-\tau \leq \xi \leq \frac{\tau}{2}\right\}}+\frac{\cos (t \tau)\{\cos (t \xi)-\cos (t \tau)\}}{\tau^{2}(\xi-\tau)(\xi+\tau)} \mathbf{1}_{\{-\tau<\xi<\tau\}} .
$$

On the one hand, it is easy to check that for all $\tau>1, \xi \in(-1,1)$ and $\varepsilon>0$,

$$
\left|\Lambda_{t}^{2}(\tau \xi, \tau)\right| \lesssim \frac{1}{\tau^{4-\varepsilon}}\left[1+\frac{1}{|1-| \xi||^{1-\varepsilon}}\right]
$$


and so

$$
\left|\int_{-\tau}^{\tau} d \xi \frac{\Lambda_{t}^{2}(\xi, \tau)}{|\xi|^{2 H_{0}-1}}\right|=\frac{1}{\tau^{2 H_{0}-2}}\left|\int_{-1}^{1} d \xi \frac{\Lambda_{t}^{2}(\tau \xi, \tau)}{|\xi|^{2 H_{0}-1}}\right| \lesssim \frac{1}{\tau^{2 H_{0}+2-\varepsilon}}
$$

On the other hand,

$$
\begin{aligned}
& \int_{-\tau}^{\tau} d \xi \frac{\Lambda_{t}^{1}(\xi, \tau)}{|\xi|^{2 H_{0}-1}}=\frac{1}{\tau^{2 H_{0}+2}} \int_{\frac{1}{2}}^{1} \frac{d \xi}{|\xi|^{2 H_{0}-1}} \frac{1-\cos (t \tau(1-\xi))}{(1-\xi)^{2}} \\
& \quad=\frac{t}{\tau^{2 H_{0}+1}} \int_{0}^{\frac{t \tau}{2}} \frac{d \xi}{\left|1-\frac{\xi}{t \tau}\right|^{2 H_{0}-1}} \frac{1-\cos \xi}{\xi^{2}} \\
& \quad=\frac{t}{\tau^{2 H_{0}+1}} \int_{0}^{\infty} d \xi \frac{1-\cos \xi}{\xi^{2}}+\frac{t}{\tau^{2 H_{0}+1}}\left[\int_{0}^{\frac{t \tau}{2}} \frac{d \xi}{\left|1-\frac{\xi}{t \tau}\right|^{2 H_{0}-1}} \frac{1-\cos \xi}{\xi^{2}}-\int_{0}^{\infty} d \xi \frac{1-\cos \xi}{\xi^{2}}\right] .
\end{aligned}
$$

The conclusion now follows immediately from the result of [1, Lemma 2.5].

\section{References}

[1] A. Deya: A non-linear wave equation with fractional perturbation. To appear in Ann. Probab.

[2] J. Ginibre and G. Velo: Generalized Strichartz inequalities for the wave equation, J. Funct. Anal. 133 (1995), 50-68.

[3] M. Gubinelli, H. Koch and T. Oh: Renormalization of the two-dimensional stochastic non linear wave equations. To appear in Trans. Amer. Math. Soc.

[4] M. Gubinelli, H. Koch and T. Oh: Paracontrolled approach to the three-dimensional stochastic nonlinear wave equation with quadratic nonlinearity. Arxiv preprint (2018).

[5] M. Hairer and C. Labbé: Multiplicative stochastic heat equations on the whole space. To appear in J. Eur. Math. Soc.

[6] M. Hairer and E. Pardoux: A Wong-Zakai theorem for stochastic PDEs. Jour. Math. Soc. Japan 67 (2015), no. 4, 15511604.

[7] J.-C. Mourrat and H. Weber: The dynamic $\varphi_{3}^{4}$ model comes down from infinity. To appear in Comm. Math. Phys..

[8] T. Runst and W. Sickel: Sobolev Spaces of Fractional Order, Nemytskij Operators, and Nonlinear Partial Differential Equations. de Gruyter Series in Nonlinear Analysis and Applications 3, Berlin (1996). 